\title{
On the Hénon equation: asymptotic profile of ground states, I
}

\section{Sur de l'équation d'Hénon: les caractéristiques limites des fonctions minimisantes, I}

\author{
Jaeyoung Byeon $^{\mathrm{a}, *}$, Zhi-Qiang Wang ${ }^{\mathrm{b}}$ \\ a Department of Mathematics, POSTECH, San 31 Hyoja-dong, Nam-gu, Pohang, Kyungbuk, 790-784, Republic of Korea \\ $\mathrm{b}^{\mathrm{b}}$ Department of Mathematics and Statistics, Utah State University, Logan, UT 84322, USA
}

Received 15 May 2002; accepted 20 April 2006

Available online 25 September 2006

\begin{abstract}
This paper is concerned with the qualitative property of the ground state solutions for the Hénon equation. By studying a limiting equation on the upper half space $\mathbf{R}_{+}^{N}$, we investigate the asymptotic energy and the asymptotic profile of the ground states for the Hénon equation. The limiting problem is related to a weighted Sobolev type inequality which we establish in this paper.

๑ 2006 L'Association Publications de l'Institut Henri Poincaré. Published by Elsevier B.V. All rights reserved

\section{Résumé}

Nous nous intéresserons, dans cet article, aux propriétés qualitatives des fonctions minimisantes (ou «ground state solutions») de l'équation d'Hénon. L'étude d'une équation limite dans le demi-espace supérieur $\mathbf{R}_{+}^{N}$, nous renseignera sur l'énergie et les caractéristiques limites des fonctions minimisantes de l'équation d'Hénon. Notons que le problème limite est en relation avec une inégalité de Sobolev pondérée que nous établirons également.
\end{abstract}

๑ 2006 L'Association Publications de l'Institut Henri Poincaré. Published by Elsevier B.V. All rights reserved

Keywords: Symmetry breaking; Minimal energy solutions; Asymptotic behaviour

\section{Introduction}

In this paper we investigate the Hénon equation [9]

$$
\Delta u+|x|^{\alpha} u^{p}=0, \quad u>0 \quad \text { in } \Omega, \quad u=0 \quad \text { on } \partial \Omega,
$$

where $\Omega$ is a bounded domain in $\mathbf{R}^{N}$. For $\alpha \geqslant 0,2<p+1<2^{*}:=\frac{2 N}{N-2}$ (in the case $N=1,2,2^{*}=+\infty$ ), it is easy to show that

\footnotetext{
* Corresponding author.

E-mail address: jbyeon@ postech.ac.kr (J. Byeon).
} 


$$
I^{\mathrm{all}, \alpha}(\Omega) \equiv \inf _{u \in H_{0}^{1}(\Omega) \backslash\{0\}} \frac{\int_{\Omega}|\nabla u|^{2} \mathrm{~d} x}{\left(\int_{\Omega}|x|^{\alpha}|u|^{p+1} \mathrm{~d} x\right)^{\frac{2}{p+1}}}
$$

is achieved by a positive function $u$, which by re-scaling gives a ground state solution of $(1) . I^{\text {all }, \alpha}(\Omega)$ is called the ground state energy, or the least energy. Numerical computations [6] suggest that when $\Omega$ is the unit ball $B(0,1)$, for some parameter $\alpha$ the ground state solutions are nonradial. This was confirmed recently in an interesting paper [14], in which it was proved that for each $2<p+1<2^{*}$ and $N \geqslant 2$, there exists $\alpha^{*}$ such that for $\alpha>\alpha^{*}$ the ground states are nonradial. In fact, the authors there compared $I^{\text {all, } \alpha}(B(0,1))$ with another minimization problem

$$
I^{\mathrm{rad}, \alpha}(B(0,1)) \equiv \inf _{u \in H_{0}^{1}(B(0,1)) \backslash\{0\}, u(x)=u(|x|)} \frac{\int_{B(0,1)}|\nabla u|^{2} \mathrm{~d} x}{\left(\int_{B(0,1)}|x|^{\alpha}|u|^{p+1} \mathrm{~d} x\right)^{\frac{2}{p+1}}} .
$$

It was shown that, if $p \in(1,(N+2) /(N-2))$ and $N \geqslant 2$, for sufficiently large $\alpha>0$,

$$
I^{\mathrm{all}, \alpha}(B(0,1))<I^{\mathrm{rad}, \alpha}(B(0,1)) .
$$

More precisely, for $N \geqslant 2$, they showed that

$$
\lim _{\alpha \rightarrow \infty}\left(\frac{N}{\alpha+N}\right)^{\frac{p+3}{p+1}} I^{\mathrm{rad}, \alpha}(B(0,1)) \in(0, \infty),
$$

and that for some $c>0$, as $\alpha \rightarrow \infty$

$$
I^{\text {all }, \alpha}(B(0,1)) \leqslant c \alpha^{2-N+\frac{2 N}{p+1}} .
$$

Our main interest in this paper is about the asymptotic profiles of both the nonradial ground state solutions and the radial ground states. This is a natural question along the line of the study and has not been addressed at all. In order to study the asymptotic profile of the ground state solutions we need to develop finer estimates on the ground state energy than those given above and to derive a limiting equation for the problem, which is essential to determining the asymptotic shape of the ground states. It turns out that the following minimization problem serves as the limiting problem for Eq. (1):

$$
J_{N, \beta}(\Omega) \equiv \inf \left\{\int_{\Omega}|\nabla u|^{2} \mathrm{~d} x \mid \int_{\Omega} \exp (-\beta t) u^{p+1} \mathrm{~d} t \mathrm{~d} y=1, u \in H(\Omega)\right\},
$$

where $\Omega=(0, \infty) \times \mathbf{R}^{N-1}, H(\Omega)$ is the completion of $C_{0}^{\infty}(\Omega)$ with respect to the norm $\|u\|^{2}=\int_{\Omega}|\nabla u|^{2} \mathrm{~d} x$, and $\beta>0$ and $N$ is the dimension. More precisely, we shall prove that for any $N \geqslant 1$ and $p>1$

$$
\lim _{\alpha \rightarrow \infty}\left(\frac{N}{\alpha+N}\right)^{\frac{p+3}{p+1}} I^{\mathrm{rad}, \alpha}(B(0,1))=\left|S^{N-1}\right|^{(p-1) /(p+1)} J_{1, N},
$$

and that for any $N \geqslant 1$ and $p \in\left(1,2^{*}-1\right)$

$$
\lim _{\alpha \rightarrow \infty}\left(\frac{N}{\alpha+N}\right)^{\frac{N+2-(N-2) p}{p+1}} I^{\text {all, } \alpha}(B(0,1))=J_{N, N} .
$$

Furthermore, through a more delicate analysis, we find asymptotic profiles of the minimizers of $I^{\text {all, } \alpha}(B(0,1))$ and $I^{\mathrm{rad}, \alpha}(B(0,1))$ as $\alpha \rightarrow \infty$. Roughly speaking, under suitable transformations the minimizers of $I^{\text {all, } \alpha}(B(0,1))$ converge to the minimizer of $J_{N, N}(\Omega)$ and the minimizers of $I^{\mathrm{rad}, \alpha}(B(0,1))$ converge to the minimizer of $J_{1, N}(\Omega)$. The precise statements will be given in Sections 3 and 4. As a byproduct of our analysis, we show that symmetry breaking occurs for all $N \geqslant 1$. On the other hand, in order to study the limiting equations, in Section 2 we first establish some weighted Sobolev type inequalities in the half space $\mathbf{R}_{+}^{N}$. These inequalities should be of independent interest of their own.

In recent years, extensive work have been done for analyzing limiting profiles of least energy solutions of singularly perturbed elliptic problems including elliptic Dirichlet, Neumann boundary value problems and nonlinear Schrödinger equations in $\mathbf{R}^{N}$. Symmetry breaking of ground state solutions has been observed for some of these problems where 
the problems are radially invariant. Most of these problems have an associated limiting problem which are usually of the following form [1]

$$
-\Delta u+u=f(u) \text { in } \mathbf{R}^{N} .
$$

The existence and uniqueness of the ground state solutions for the limiting problems are used to get information for the ground state solutions of the singularly perturbed problems. For Hénon equation (1) we see that the appropriate limiting equation is more complicated. The analysis of the limiting equation will be done in Section 2 . After getting information for the limiting problem we shall study the asymptotic property of both the radial ground state solutions and nonradial ground state solutions of the Hénon equation (1). These are done in Sections 3 and 4, respectively.

\section{Limiting equations and a weighted Sobolev type inequality}

As we mentioned, by a suitable transformation of the ground states, we obtain, in Section 3, the following limiting problem for Eq. (1).

$$
\begin{aligned}
& \Delta u+\exp (-\beta t) u^{p}=0, \quad u>0 \quad \text { in }(0, \infty) \times \mathbf{R}^{N-1}, \\
& u=0 \quad \text { on } \partial\left((0, \infty) \times \mathbf{R}^{N-1}\right),
\end{aligned}
$$

where $\beta>0$.

In this section, we shall first study this equation. In fact, we shall work on more general situations and consider more general domain $\Omega$ than $(0, \infty) \times \mathbf{R}^{N-1}$. Let $(t, y) \in(-\infty, \infty) \times \mathbf{R}^{N-1}$. Let $\Omega$ be a domain in $(-\infty, \infty) \times \mathbf{R}^{N-1}$. Throughout this section, we assume that there exists $L>0$ such that

$$
\Omega \subset(-L, \infty) \times \mathbf{R}^{N-1} .
$$

It is well known that there exists no solution for

$$
\Delta u+u^{p}=0, \quad u>0 \quad \text { in }(0, \infty) \times \mathbf{R}^{N-1}, \quad u=0 \quad \text { on }\{0\} \times \mathbf{R}^{N-1} .
$$

Moreover, for $p \in[1,(N+2) /(N-2))$,

$$
\sup _{\varphi \in C_{0}^{\infty}\left((0, \infty) \times \mathbf{R}^{N-1}\right) \backslash\{0\}} \frac{\left(\int_{(0, \infty) \times \mathbf{R}^{N-1}}|\varphi|^{p+1} \mathrm{~d} s \mathrm{~d} y\right)^{2 /(p+1)}}{\int_{(0, \infty) \times \mathbf{R}^{N-1}}|\nabla \varphi|^{2} \mathrm{~d} s \mathrm{~d} y}=\infty .
$$

On the other hand, we have the following weighted Sobolev inequality.

Proposition 2.1. Let $p \in[1,(N+2) /(N-2)]$ for $N \geqslant 3, p \in[1, \infty)$ for $N=1,2$ and $\beta>0$. Then, there exists a constant $C>0$, depending only on $\beta, p, N$ and $L$, such that for any $\varphi \in C_{0}^{\infty}(\Omega)$,

$$
\left(\int_{\Omega} \exp (-\beta t)|\varphi|^{p+1} \mathrm{~d} s \mathrm{~d} y\right)^{\frac{2}{p+1}} \leqslant C \int_{\Omega}|\nabla \varphi|^{2} \mathrm{~d} s \mathrm{~d} y .
$$

Proof. Since $\exp (-\beta t) \leqslant \exp (\beta L)$ in $\Omega$, the case $p=(N+2) /(N-2)$ for $N \geqslant 3$ comes from Sobolev inequality.

Let $\varphi \in C_{0}^{\infty}(\Omega)$. We see from integration by parts and Cauchy's inequality that

$$
\begin{aligned}
\int_{-\infty}^{\infty} \exp (-\beta t)(\varphi(y, t))^{2} \mathrm{~d} t & =\frac{2}{\beta} \int_{-\infty}^{\infty} \exp (-\beta t) \varphi(y, t) \varphi_{t}(y, t) \mathrm{d} t \\
& \leqslant \frac{1}{2} \int_{-\infty}^{\infty} \exp (-\beta t)(\varphi(y, t))^{2} \mathrm{~d} t+\frac{8}{\beta^{2}} \int_{-\infty}^{\infty} \exp (-\beta t)\left(\varphi_{t}(y, t)\right)^{2} \mathrm{~d} t .
\end{aligned}
$$

Then, it follows that

$$
\int_{-\infty}^{\infty} \exp (-\beta t)(\varphi(y, t))^{2} \mathrm{~d} t \leqslant \frac{16}{\beta^{2}} \int_{-\infty}^{\infty} \exp (-\beta t)\left(\varphi_{t}(y, t)\right)^{2} \mathrm{~d} t .
$$


Thus, by integrating both sides over $\mathbf{R}^{N-1}$ in above inequality, we deduce that for any $\varphi \in C_{0}^{\infty}(\Omega)$,

$$
\begin{aligned}
\int_{\Omega} \exp (-\beta t)(\varphi(y, t))^{2} \mathrm{~d} s \mathrm{~d} y & \leqslant \frac{16}{\beta^{2}} \int_{\Omega} \exp (-\beta t)\left(\varphi_{t}(y, t)\right)^{2} \mathrm{~d} s \mathrm{~d} y \\
& \leqslant \frac{16}{\beta^{2}} \exp (\beta L) \int_{\Omega}|\nabla \varphi(y, t)|^{2} \mathrm{~d} s \mathrm{~d} y .
\end{aligned}
$$

This proves the case $p=1$.

From now on, we assume that $p \in\left(1,2^{*}-1\right)$.

Let $N \geqslant 3$. Since $p+1 \in(2,2 N /(N-2))$, there exists $s \in(0,1)$ such that $p+1=2 s+(1-s) 2 N /(N-2)$. Then, from Hölder's inequality, we see that

$$
\begin{aligned}
\int_{\Omega} \exp (-\beta t)(\varphi(y, t))^{p+1} \mathrm{~d} s \mathrm{~d} y & \leqslant\left(\int_{\Omega} \exp (-\beta t) \phi^{2} \mathrm{~d} s \mathrm{~d} y\right)^{s}\left(\int_{\Omega} \exp (-\beta t) \phi^{2 N /(N-2)} \mathrm{d} s \mathrm{~d} y\right)^{1-s} \\
& \leqslant \exp (\beta L)\left(\int_{\Omega} \exp (-\beta t) \varphi^{2} \mathrm{~d} s \mathrm{~d} y\right)^{s}\left(\int_{\Omega} \varphi^{2 N /(N-2)} \mathrm{d} s \mathrm{~d} y\right)^{1-s} .
\end{aligned}
$$

Then, by Sobolev inequalities, there exists a constant $C>0$ that $\varphi \in C_{0}^{\infty}(\Omega)$,

$$
\int_{\Omega} \exp (-\beta t)(\varphi(y, t))^{p+1} \mathrm{~d} s \mathrm{~d} y \leqslant C\left(\int_{\Omega}|\nabla \varphi(y, t)|^{2} \mathrm{~d} s \mathrm{~d} y\right)^{(p+1) / 2} .
$$

Thus, the case of $N \geqslant 3$ is finished.

For the case $N=1$, we see that

$$
\exp \left(-\frac{\beta t}{2(p-1)}\right) \varphi(t)=\int_{-\infty}^{t}-\frac{\beta}{2(p-1)} \exp \left(-\frac{\beta s}{2(p-1)}\right) \varphi(s)+\exp \left(-\frac{\beta s}{2(p-1)}\right) \varphi^{\prime}(s) \mathrm{d} s .
$$

Thus, it follows from Cauchy's inequality and (7) that for some $C=C(p, \beta, L)>0$,

$$
\begin{aligned}
\sup _{t \in(-\infty, \infty)}\left|\exp \left(-\frac{\beta t}{2(p-1)}\right) \varphi(t)\right|^{2} \leqslant & \left(\frac{\beta}{2(p-1)}\right)^{2} \int_{-L}^{\infty} \exp \left(-\frac{\beta s}{2(p-1)}\right) \mathrm{d} s \int_{-\infty}^{\infty} \exp \left(-\frac{\beta s}{2(p-1)}\right)(\varphi(s))^{2} \mathrm{~d} s \\
& +\int_{-L}^{\infty} \exp \left(-\frac{\beta s}{2(p-1)}\right) \mathrm{d} s \int_{-\infty}^{\infty} \exp \left(-\frac{\beta s}{2(p-1)}\right)\left(\varphi^{\prime}(s)\right)^{2} \mathrm{~d} s \\
\leqslant & C \int_{-\infty}^{\infty}\left(\varphi^{\prime}(s)\right)^{2} \mathrm{~d} s .
\end{aligned}
$$

Then, for any $p \geqslant 1$, we deduce from (5) that for some $C=C(\beta, p, L)>0$,

$$
\begin{aligned}
\int_{-\infty}^{\infty} \exp (-\beta t)(\varphi(t))^{p+1} \mathrm{~d} t & \leqslant \sup _{t \in(-\infty, \infty)}\left|\exp \left(-\frac{\beta t}{2(p-1)}\right) \varphi(t)\right|^{p-1} \int_{-\infty}^{\infty} \exp \left(-\frac{\beta t}{2}\right)(\varphi(t))^{2} \mathrm{~d} t \\
& \leqslant C\left(\int_{-\infty}^{\infty}\left(\varphi^{\prime}\right)^{2} \mathrm{~d} t\right)^{(p+1) / 2} .
\end{aligned}
$$

For the case $N=2$, we see that 


$$
\begin{aligned}
& \exp \left(-\frac{\beta}{2} t\right)|\varphi(y, t)| \leqslant \int_{-\infty}^{y} \exp \left(-\frac{\beta}{2} t\right)\left|\frac{\partial \varphi(y, t)}{\partial y}\right| \mathrm{d} y \\
& \exp \left(-\frac{\beta}{2} t\right)|\varphi(y, t)| \leqslant \int_{-\infty}^{t} \exp \left(-\frac{\beta}{2} t\right)\left|\frac{\partial \varphi(y, t)}{\partial t}\right|+\frac{\beta}{2} \exp \left(-\frac{\beta}{2} t\right)|\varphi(y, t)| \mathrm{d} t .
\end{aligned}
$$

Then, multiplying each sides and integrating over $\Omega$, we see that

$$
\begin{aligned}
\int_{\Omega} \exp (-\beta t)(\varphi(y, t))^{2} \mathrm{~d} t \mathrm{~d} y \leqslant & \left(\int_{\Omega} \exp \left(-\frac{\beta}{2} t\right)|\nabla \varphi(y, t)| \mathrm{d} s \mathrm{~d} y\right)^{2} \\
& +\frac{\beta}{2} \int_{\Omega} \exp \left(-\frac{\beta}{2} t\right)|\nabla \varphi(y, t)| \mathrm{d} s \mathrm{~d} y \int_{\Omega} \exp \left(-\frac{\beta}{2} t\right)|\varphi(y, t)| \mathrm{d} t \mathrm{~d} y .
\end{aligned}
$$

Replacing $\varphi$ by $\varphi^{m}$ in above inequality, we deduce from Cauchy's inequality that

$$
\begin{aligned}
\int_{\Omega} \exp (-\beta t) \varphi^{2 m} \leqslant & \left(\int_{\Omega} \exp \left(-\frac{\beta}{2} t\right) m|\varphi|^{m-1}|\nabla \varphi|\right)^{2}+\frac{\beta}{2} \int_{\Omega} \exp \left(-\frac{\beta}{2} t\right) m|\varphi|^{m-1}|\nabla \varphi| \int_{\Omega} \exp \left(-\frac{\beta}{2} t\right)|\varphi|^{m} \\
\leqslant & m^{2} \int_{\Omega} \exp \left(-\frac{\beta}{2} t\right) \varphi^{2(m-1)} \int_{\Omega} \exp \left(-\frac{\beta}{2} t\right)|\nabla \varphi|^{2} \\
& +\frac{m \beta}{2}\left(\int_{\Omega} \exp \left(-\frac{\beta}{2} t\right) \varphi^{2(m-1)} \int_{\Omega} \exp \left(-\frac{\beta}{2} t\right)|\nabla \varphi|^{2}\right)^{\frac{1}{2}} \int_{\Omega} \exp \left(-\frac{\beta}{2} t\right)|\varphi|^{m} .
\end{aligned}
$$

Thus, there exists $C=C(m, \beta, L)>0$ such that for any $\varphi \in C_{0}^{\infty}(\Omega)$,

$$
\begin{aligned}
\int_{\Omega} \exp (-\beta t) \varphi^{2 m} \leqslant & C \int_{\Omega} \exp \left(-\frac{\beta}{2} t\right) \varphi^{2(m-1)} \int_{\Omega}|\nabla \varphi|^{2} \\
& +\frac{m \beta}{2}\left(\int_{\Omega} \exp \left(-\frac{\beta}{2} t\right) \varphi^{2(m-1)} \int_{\Omega}|\nabla \varphi|^{2}\right)^{\frac{1}{2}} \int_{\Omega} \exp \left(-\frac{\beta}{2} t\right)|\varphi|^{m} .
\end{aligned}
$$

Then, if it holds that for some $C=C(m, \beta, L)>0$,

$$
\left(\int_{\Omega} \exp \left(-\frac{\beta}{2} t\right)|\varphi|^{m}\right)^{2 / m} \leqslant C \int_{\Omega}|\nabla \varphi|^{2},
$$

and

$$
\left(\int_{\Omega} \exp \left(-\frac{\beta}{2} t\right) \varphi^{2(m-1)}\right)^{1 /(m-1)} \leqslant C \int_{\Omega}|\nabla \varphi|^{2},
$$

it follows that for some $C=C(m, \beta, L)>0$,

$$
\left(\int_{\Omega} \exp (-\beta t) \varphi^{2 m}\right)^{1 / m} \leqslant C \int_{\Omega}|\nabla \varphi|^{2} .
$$

Note from (5) that (8) and (9) hold for $m=2$. Therefore, we deduce by induction that (10) holds for any $m \geqslant 1$. This completes the proof. 
We define $H(\Omega)$ to be the completion of $C_{0}^{\infty}(\Omega)$ with respect to the following norm

$$
\|u\| \equiv\left(\int_{\Omega}|\nabla u|^{2} \mathrm{~d} t \mathrm{~d} y\right)^{1 / 2} .
$$

Remark 2.1. We note that, due to Sobolev embedding, $H(\Omega)=D_{0}^{1,2}(\Omega)$ is well defined for $N \geqslant 3$ and any domain $\Omega \subset \mathbf{R}^{N}$. Proposition 2.1 assures that the space $H(\Omega)$ is also well defined as a space of functions for $N=1,2$ if there exists $L>0$ such that $\Omega \subset(-L, \infty) \times \mathbf{R}^{N-1}$.

Now, we are interested in the existence of a ground state solution of (2). As we will see in the following, the existence depends on the shape of $\partial \Omega$. The following condition (referred as E-condition later) is a natural one, in a technical reason, for the existence of a ground state solution. The existence will be given in Proposition 2.3. We do not know whether this condition is optimal or not (see Proposition 2.7 and [3] for related results).

Definition 2.2. We say that $\Omega$ satisfies E-condition if there exists a fixed point $y^{0}=\left(y_{1}^{0}, \ldots, y_{N-1}^{0}\right) \in \mathbf{R}^{N-1}$ such that for any $\left(t, y_{1}, \ldots, y_{N-1}\right) \in \Omega$ and $s_{1}, \ldots, s_{N-1} \in[-1,1]$,

$$
\left(t, y_{1}^{0}+s_{1}\left|y_{1}-y_{1}^{0}\right|, \ldots, y_{N-1}^{0}+s_{N-1}\left|y_{N-1}-y_{N-1}^{0}\right|\right) \in \Omega,
$$

or if for each $T>0, \Omega \cap\left((-L, T) \times \mathbf{R}^{N-1}\right)$ is bounded.

Proposition 2.3. Let $p \in\left(1,2^{*}-1\right)$ and $\beta>0$. Suppose that a domain $\Omega$ satisfies E-condition. Then, there exists a minimizer $u \in H(\Omega)$ of the following minimization problem

$$
J_{N, \beta}(\Omega) \equiv \inf \left\{\|u\|^{2} \mid \int_{\Omega} \exp (-\beta t) u^{p+1} \mathrm{~d} t \mathrm{~d} y=1, u \in H(\Omega)\right\} .
$$

To prove Proposition 2.3, we need some preparation. We first consider an eigenvalue problem

$$
\begin{aligned}
& \frac{\mathrm{d}^{2} \phi}{\mathrm{d} t^{2}}+\lambda \exp (-\beta t) \phi=0 \quad \text { on }(0, \infty), \\
& \phi(0)=0, \\
& \phi \in H((0, \infty)) .
\end{aligned}
$$

Let $s=\frac{2 \sqrt{\lambda}}{\beta} \exp \left(-\frac{\beta}{2} t\right)$ and $w(s)=\phi(t)$. Then, it follows that

$$
\frac{\mathrm{d}^{2} w}{\mathrm{~d} s^{2}}+\frac{1}{s} w_{s}+w=0
$$

Note that for some $C>0$,

$$
|\phi(t)|=\left|\int_{0}^{t} \phi^{\prime}(s) \mathrm{d} s\right| \leqslant \sqrt{t}\left(\int_{0}^{\infty}\left|\phi^{\prime}\right|^{2} \mathrm{~d} t\right)^{1 / 2} \leqslant C \sqrt{t} .
$$

This implies that for some $C>0$,

$$
|w(s)| \leqslant C(1+|\log s|)^{1 / 2} .
$$

There are two kinds of solutions, Bessel functions of the first kind $J_{0}$ and the second kind $N_{0}$, for $(12)$. Since $N_{0}(s) \cong$ $\log s$ near 0 , it follows that $w(s)=J_{0}(s)$. The Bessel function of the first kind $J_{0}(s)$ is given by $\sum_{n=0}^{\infty} \frac{(-1)^{n}}{(n !)^{2}}\left(\frac{s}{2}\right)^{2 n}$. Let $j_{1}<j_{2}<\cdots$ be the positive zeros of $J_{0}$. Then, it is well known that

$$
J_{0}(s)=\prod_{n=1}^{\infty}\left(1-\frac{s^{2}}{\left(j_{n}\right)^{2}}\right) .
$$

Thus, we have the following lemma. 
Lemma 2.4. The eigenvalues $\left\{\lambda_{\beta, n}\right\}_{n=1}^{\infty}$ of (11) are given by

$$
\lambda_{\beta, n}=\frac{\left(j_{n}\right)^{2} \beta^{2}}{4}, \quad n=1,2, \ldots
$$

The eigenfunction $\phi_{\beta, n}$ corresponding to $\lambda_{\beta, n}$ is given by

$$
\phi_{\beta, n}(t)=J_{0}\left(\frac{2 \sqrt{\lambda_{\beta, n}}}{\beta} \exp \left(-\frac{\beta}{2} t\right)\right), \quad t \in[0, \infty) .
$$

Proof of Proposition 2.3. Let $\left\{v_{n}\right\}_{n}$ be a minimizing sequence of $J_{N, \beta}(\Omega)$. Since $H(\Omega)$ is the completion of $C_{0}^{\infty}(\Omega)$, we can assume that $\left\{v_{n}\right\}_{n} \subset C_{0}^{\infty}(\Omega)$. We take $T_{n}^{1}, T_{n}^{2}>0$ such that for each positive integer $n$,

$$
\operatorname{supp}\left(v_{n}\right) \subset\left\{(t, y) \in \Omega\left|-L<t<T_{n}^{1},\right| y \mid<T_{n}^{2}\right\},
$$

and that for each $i=1,2, T_{1}^{i}<T_{2}^{i}<\cdots$ and $\lim _{n \rightarrow \infty} T_{n}^{i}=\infty$. Define

$$
D_{n} \equiv\left(-L, T_{n}^{1}\right) \times\left\{y \in \mathbf{R}^{N-1}|| y \mid \leqslant T_{n}^{2}\right\} .
$$

When $\Omega \cap\left(\left(-L, T_{n}^{1}\right) \times \mathbf{R}^{N-1}\right)$ is bounded, we can take larger $T_{n}^{2}$ so that $\Omega \cap\left(\left(-L, T_{n}^{1}\right) \times \mathbf{R}^{N-1}\right) \subset D_{n}$. Then, we consider a following minimization problem

$$
I_{n} \equiv \inf \left\{\|u\|^{2} \mid \int_{\Omega \cap D_{n}} \exp (-\beta t) u^{p+1} \mathrm{~d} t \mathrm{~d} y=1, u \in H_{n} \backslash\{0\}\right\},
$$

where $H_{n} \equiv H_{0}^{1,2}\left(\Omega \cap D_{n}\right)$. Since $D_{n}$ is bounded, there exists a nonnegative minimizer $u_{n}$ of $I_{n}$ for each $n \geqslant 1$. It is easy to see that $I_{n} \rightarrow J_{N, \beta}(\Omega)$ as $n \rightarrow \infty$.

When $\Omega \cap\left(\left(-L, T_{n}^{1}\right) \times \mathbf{R}^{N-1}\right)$ is not bounded, from a Steiner symmetrization (refer [10]), we can assume that $u_{n}\left(t, y^{0}+z\right)$ is even with respect to each of the components of $z=\left(z_{1}, \ldots, z_{N}-1\right)$ and is monotone decreasing in each of the components of $z$. Then, $\left\{u_{n}\right\}_{n}$ is also a minimizing sequence of $J_{N, \beta}(\Omega)$, and $\lim _{n \rightarrow \infty} I_{n}=J_{N, \beta}(\Omega)$.

Moreover, we see that

$$
\begin{array}{ll}
\Delta u_{n}+I_{n} \exp (-\beta t)\left(u_{n}\right)^{p}=0 & \text { in } \Omega \cap D_{n}, \\
u_{n}>0 & \text { in } \Omega \cap D_{n}, \\
u_{n}=0 & \text { on } \partial\left(\Omega \cap D_{n}\right) .
\end{array}
$$

Note that

$$
\begin{aligned}
& \Delta u_{n}+I_{n} \exp (-\beta L)\left(u_{n}\right)^{p} \geqslant 0 \quad \text { in } \Omega \cap D_{n}, \\
& u_{n}=0 \quad \text { on } \partial\left(\Omega \cap D_{n}\right) .
\end{aligned}
$$

Then, since $\left\{\left\|u_{n}\right\|_{\left.L^{2 N /(N-2)}\right\}_{n}}\right.$ is bounded for $N \geqslant 3$, from a uniform $L^{\infty}$-estimate [2, Proposition 3.5], we see that $\left\{\left\|u_{n}\right\|_{L^{\infty}}\right\}_{n}$ is bounded for $N \geqslant 3$. For $N=1$, it follows that for some $C>0$,

$$
u_{n}(t)=\int_{-L}^{t} u_{n}^{\prime}(s) \mathrm{d} s \leqslant \sqrt{t+L}\left(\int_{-L}^{\infty}\left|u_{n}^{\prime}(s)\right|^{2} \mathrm{~d} s\right)^{1 / 2} \leqslant C \sqrt{t} .
$$

For $N=2$, we use the Green function on the upper half plane. Then there is a constant $C>0$,

$$
u(t-L, x) \leqslant C \int_{0}^{\infty} \int_{-\infty}^{\infty} \log \frac{(t+s)^{2}+(x-y)^{2}}{(t-s)^{2}+(x-y)^{2}} \exp (-\beta s) u^{p}(y) \mathrm{d} y \mathrm{~d} s .
$$

Since $\left\{\int_{0}^{\infty} \int_{-\infty}^{\infty} \exp (-\beta s)\left(u_{n}\right)^{p+1}(y) \mathrm{d} y \mathrm{~d} s\right\}_{n}$ is bounded, from Hölder's inequality, it follows that for some $C>0$, 


$$
\begin{aligned}
\left(u_{n}(t-L, x)\right)^{p+1} & \leqslant C \int_{0}^{\infty} \int_{-\infty}^{\infty}\left(\log \frac{(t+s)^{2}+(x-y)^{2}}{(t-s)^{2}+(x-y)^{2}}\right)^{p+1} \exp (-\beta s) \mathrm{d} y \mathrm{~d} s \\
& \leqslant C \exp (-\beta t) \int_{-t}^{\infty} \int_{-\infty}^{\infty}\left(\log \frac{(s+2 t)^{2}+(x-y)^{2}}{s^{2}+(x-y)^{2}}\right)^{p+1} \exp (-\beta s) \mathrm{d} y \mathrm{~d} s \\
& =C \exp (-\beta t) \int_{-t}^{\infty} \int_{-\infty}^{\infty}\left(\log \left(1+\frac{4 t(s+t)}{s^{2}+(x-y)^{2}}\right)\right)^{p+1} \exp (-\beta s) \mathrm{d} y \mathrm{~d} s \\
& =C \exp (-\beta t) \int_{-t}^{\infty} \int_{-\infty}^{\infty}\left(\log \left(1+\frac{4 t(s+t)}{s^{2}+y^{2}}\right)\right)^{p+1} \exp (-\beta s) \mathrm{d} y \mathrm{~d} s .
\end{aligned}
$$

Note that $\log (1+a) \leqslant a$ for $a>0$. Thus, for some constant $C>0$ and $t>0$,

$$
\begin{aligned}
\int_{-t}^{\infty} & \int_{-\infty}^{\infty}\left(\log \left(1+\frac{4 t(s+t)}{s^{2}+y^{2}}\right)\right)^{p+1} \exp (-\beta s) \mathrm{d} y \mathrm{~d} s \\
= & \int_{(-t, \infty) \times(-\infty, \infty) \cap B(0,1)}\left(\log \left(1+\frac{4 t(s+t)}{s^{2}+y^{2}}\right)\right)^{p+1} \exp (-\beta s) \mathrm{d} y \mathrm{~d} s \\
& +\int_{(-t, \infty) \times(-\infty, \infty) \backslash B(0,1)}\left(\log \left(1+\frac{4 t(s+t)}{s^{2}+y^{2}}\right)\right)^{p+1} \exp (-\beta s) \mathrm{d} y \mathrm{~d} s \\
\leqslant & \int_{0}^{1} \int_{0}^{2 \pi}\left(\log \left(1+\frac{4 t(1+t)}{r^{2}}\right)\right)^{p+1} \exp (-\beta r \sin \theta) r \mathrm{~d} \theta \mathrm{d} r+(4 t)^{p+1} \int_{-1}^{1} \int_{s=\sqrt{1+y^{2}}}^{\infty}(s+t)^{p+1} \exp (-\beta s) \mathrm{d} s \mathrm{~d} y \\
& +(4 t)^{p+1} \int_{-1}^{1} \int_{s=-t}^{\infty}(s+t)^{p+1} \exp (-\beta s) \mathrm{d} s \mathrm{~d} y+(4 t)^{p+1} \int_{|y| \geqslant 1}^{\infty}\left(\frac{(s+t)}{y^{2}}\right)^{p+1} \exp (-\beta s) \mathrm{d} s \mathrm{~d} y \\
\leqslant & C t(1+t)+C t^{p+1} \int_{-t}^{\infty} \int_{s=-t}^{\infty}(s+t)^{p+1} \exp (-\beta s) \mathrm{d} s \\
\leqslant & C(t(1+t))^{p+1} \exp (\beta t) .
\end{aligned}
$$

This implies that there exists a constant $C>0$, independent of $n \geqslant 1$, such that

$$
u_{n}(t, x) \leqslant C(1+t)^{C}, \quad t>0 .
$$

Having established some upper bounds we need an estimate from below for the $L^{\infty}$ norm. From Proposition 2.2 and the fact that

$$
\int_{\Omega \cap D_{n}}\left|\nabla u_{n}\right|^{2} \mathrm{~d} x \mathrm{~d} t \leqslant I_{n}\left\|u_{n}\right\|_{L^{\infty}}^{p-1} \int_{\Omega \cap D_{n}} \exp (-\beta t)\left(u_{n}\right)^{2} \mathrm{~d} x \mathrm{~d} t,
$$

we see that the set $\left\{\left\|u_{n}\right\|_{L^{\infty}}\right\}_{n}$ is bounded away from 0 .

Next, we consider the convergence of $u_{n}$ and we consider several cases. First, for $N=1$, it is easy to see that

$$
\lim _{T \rightarrow \infty} \int_{T}^{\infty} \exp (-\beta t)\left(u_{n}\right)^{p+1} \mathrm{~d} t=0 \quad \text { uniformly with respect to } n=1,2, \ldots
$$


Since $\left\{u_{n}\right\}_{n}$ is bounded in $H, u_{n}$ converges weakly to some $u$ in $H$. Then,

$$
\int_{\Omega} \exp (-\beta t) u^{p+1} \mathrm{~d} t=1 .
$$

This implies that $u$ is a minimizer of $J_{1, \beta}(\Omega)$.

For the case $N \geqslant 2$, we claim that for sufficiently large $T>0$,

$$
\liminf _{n \rightarrow \infty} \sup \left\{u_{n}(t, y) \mid-L<t<T,(t, y) \in \Omega \cap D_{n}\right\}>0 .
$$

Suppose that it is not true. Then, for any $T>0$,

$$
\liminf _{n \rightarrow \infty} \sup _{-L<t<T,(t, y) \in \Omega \cap D_{n}} u_{n}(t, y)=0 .
$$

Taking a subsequence if necessary, we can assume that for sufficiently large $T>0$,

$$
\lim _{n \rightarrow \infty} \sup _{-L<t<T,(t, y) \in \Omega \cap D_{n}} u_{n}(t, y)=0 .
$$

By Lemma 2.4 we let $\left(\phi_{\frac{\beta}{2}, 1}, \lambda_{\frac{\beta}{2}, 1}\right)$ be a pair of the first eigenfunction and the first eigenvalue of

$$
\begin{aligned}
& \frac{\mathrm{d}^{2} \phi}{\mathrm{d} t^{2}}+\lambda \exp \left(-\frac{\beta t}{2}\right) \phi=0 \quad \text { on }(0, \infty), \\
& \phi(0)=0, \\
& \phi \in H((0, \infty))
\end{aligned}
$$

satisfying that for $t>0, \phi_{\frac{\beta}{2}, 1}(t)>0$, and $\lim _{t \rightarrow \infty} \phi_{\frac{\beta}{2}, 1}(t)=1$. From the estimate (14) for $N=2$ and the boundedness of $\left\{\left\|u_{n}\right\|_{L^{\infty}}\right\}_{n}$ for $N \geqslant 3$, we see that $\lim _{t \rightarrow \infty} \exp \left(-\frac{\beta}{2} t\right)\left(u_{n}(t, y)\right)^{p-1}=0$ uniformly with respect to $y \in \mathbf{R}^{N-1}$. Thus, there exists sufficiently large $T>0$ such that

$$
\Delta \phi_{\frac{\beta}{2}, 1}+I_{n} \exp (-\beta t)\left(u_{n}\right)^{p-1} \phi_{\frac{\beta}{2}, 1}=\phi_{\frac{\beta}{2}, 1} \exp (-\beta t)\left(I_{n} u_{n}^{p-1}-\exp \left(\frac{\beta t}{2}\right) \lambda_{\frac{\beta}{2}, 1}\right) \leqslant 0, \quad t \geqslant T .
$$

From a comparison principle (refer [12]), we see that for each $n=1, \ldots$, and $t \geqslant T$,

$$
u_{n}(t, y) \leqslant \phi_{\frac{\beta}{2}, 1}(t) \max _{\left\{y \mid(T, y) \in \Omega \cap \tilde{D}_{n}\right\}} \frac{u_{n}(T, y)}{\phi_{\frac{\beta}{2}, 1}(T)} .
$$

This implies that

$$
\lim _{n \rightarrow \infty} \sup _{(t, y) \in \Omega \cap D_{n}} u_{n}(t, y)=0
$$

this contradicts that $\left\{\left\|u_{n}\right\|_{L^{\infty}}\right\}_{n}$ is bounded away from 0 . This proves the claim.

Now, taking a subsequence if necessary, we can assume that $u_{n}$ converges weakly to some $u$ in $H(\Omega)$ as $n \rightarrow \infty$. From the boundedness of $\left\{\left\|u_{n}\right\|_{L^{\infty}}\right\}_{n}$, we see that for some $\rho \in(0,1),\left\{\left\|u_{n}\right\|_{C^{2, \rho}}\right\}_{n}$ is bounded. Thus, we can assume that $u_{n}$ converges locally to $u$ in $C^{2}$ as $n \rightarrow \infty$. Note that $\lim _{n \rightarrow \infty} I_{n}=J_{N, \beta}(\Omega)$. Thus, this $u$ satisfies the following equation

$$
\begin{aligned}
& \Delta u+J_{N, \beta}(\Omega) \exp (-\beta t) u^{p}=0 \quad \text { in } \Omega, \\
& u=0 \text { on } \partial \Omega .
\end{aligned}
$$

From the preceding claim, we see that if $\Omega$ satisfies the first assertion of E-condition in Definition 2.2, for sufficiently large $T>0$,

$$
\liminf _{n \rightarrow \infty} \max _{t \in(-L, T),(t, y) \in \Omega \cap D_{n}} u_{n}(t, y)=\liminf _{n \rightarrow \infty} \max _{t \in(-L, T)} u_{n}\left(t, y^{0}\right)>0,
$$

and that if $\Omega$ satisfies the second assertion of E-condition in Definition 2.2, for sufficiently large $T>0$,

$$
\liminf _{n \rightarrow \infty} \max _{-L<t<T,(t, y) \in \Omega} u_{n}(t, y)=\liminf _{n \rightarrow \infty} \max _{-L<t<T,(t, y) \in \Omega \cap D_{n}} u_{n}(t, y)>0 .
$$


Thus, it follows that $u \neq 0$. Let

$$
\gamma \equiv \int_{\Omega} \exp (-\beta t) u^{p+1} \mathrm{~d} t \mathrm{~d} y \in(0,1] .
$$

Suppose that $\gamma<1$. Then, taking $w=\gamma^{-1 /(p+1)} u$, we see that

$$
\int_{\Omega} \exp (-\beta t) w^{p+1} \mathrm{~d} t \mathrm{~d} y=1,
$$

and that

$$
\begin{aligned}
& \Delta w+J_{N, \beta}(\Omega) \gamma^{(p-1) /(p+1)} \exp (-\beta t) w^{p}=0 \text { in } \Omega, \\
& w>0 \text { in } \Omega, \\
& w=0 \text { on } \partial \Omega .
\end{aligned}
$$

By integration by parts, we get

$$
\int_{\Omega}|\nabla w|^{2} \mathrm{~d} t \mathrm{~d} y=J_{N, \beta}(\Omega) \gamma^{(p-1) /(p+1)}<J_{N, \beta}(\Omega) .
$$

This contradicts the definition of $J_{N, \beta}(\Omega)$. Thus, it follows that

$$
\int_{\Omega} \exp (-\beta t) u^{p+1} \mathrm{~d} t \mathrm{~d} y=1 .
$$

Since

$$
\int_{\Omega}|\nabla u|^{2} \mathrm{~d} t \mathrm{~d} y \leqslant \liminf _{n \rightarrow \infty} \int_{\Omega}\left|\nabla u_{n}\right|^{2} \mathrm{~d} t \mathrm{~d} y=J_{N, \beta}(\Omega),
$$

we conclude that $u$ is a minimizer of $J_{N, \beta}(\Omega)$. This completes the proof.

For our applications to the Hénon equation (1), we are particularly interested in the case $\Omega=(0, \infty) \times \mathbf{R}^{N-1}$. In this case, we derive the following qualitative properties of the minimizers of $J_{N, \beta}\left((0, \infty) \times \mathbf{R}^{N-1}\right)$.

Proposition 2.5. Let $u$ be a nonnegative minimizer of $J_{N, \beta}\left((0, \infty) \times \mathbf{R}^{N-1}\right)$. Then,

(i) For $N=1, u$ is a monotone increasing bounded function, and for any $c \in(0, \beta)$, there exists constants $C>0$ such that

$$
0<\lim _{s \rightarrow \infty} u(s)-u(t) \leqslant C \exp (-c t)
$$

(ii) For $N \geqslant 2$, for some $x_{0} \in \mathbf{R}^{N-1}, u(t, x)$ depends only on $t$ and $\left|x-x_{0}\right|$, and is monotone decreasing with respect to $\left|x-x_{0}\right|$;

(iii) For $N \geqslant 2, \lim _{|x| \rightarrow \infty} u(t, x)=0$;

(iv) For $N \geqslant 3, \lim _{|(t, x)| \rightarrow \infty} u(t, x)=0$;

(v) For $N \geqslant 4$, there exists $C>0$ independent of $t>0$ such that $u(t, x) \leqslant \frac{C}{|x|^{N-3}}$.

Proof. For simplicity of notations, let $J=J_{N, \beta}\left((0, \infty) \times \mathbf{R}^{N-1}\right)$. First of all, we note that

$$
\begin{aligned}
& \Delta u+J \exp (-\beta t) u^{p}=0, \quad u>0 \quad \text { in }(0, \infty) \times \mathbf{R}^{N-1}, \\
& u=0 \quad \text { on }\{0\} \times \mathbf{R}^{N-1} .
\end{aligned}
$$


We prove (i) first. For $N=1$, we see that

$$
u(t)=\int_{0}^{t} u^{\prime}(s) \mathrm{d} s \leqslant \sqrt{t}\left(\int_{0}^{t}\left(u^{\prime}(s)\right)^{2} \mathrm{~d} s\right)^{1 / 2} \leqslant C \sqrt{t} .
$$

Denoting $w(t)=u^{\prime}(t)$, we see that

$$
w^{\prime \prime}+\beta w^{\prime}+p J \exp (-\beta t) u^{p-1} w=0 \quad \text { in }(0, \infty) .
$$

It is easy to see that $w(0)>0$, and that $u$ is monotone increasing and $w$ is monotone decreasing. Since $\int_{0}^{\infty} w^{2}(t) \mathrm{d} t<$ $\infty$, it follows that $\lim _{t \rightarrow \infty} w(t)=0$. Since $u(t) \leqslant C \sqrt{t}$, it follows that for $\phi(t)=\exp (-c t)$ with $c \in(0, \beta)$,

$$
\phi^{\prime \prime}+\beta \phi^{\prime}+p J \exp (-\beta t) u^{p-1} \phi<0 \quad \text { in }(T, \infty)
$$

if $T>0$ is sufficiently large. By a comparison principle (refer [12]), we see that for some $C>0$,

$$
w(t) \leqslant C \exp (-c t), \quad t \in(0, \infty) .
$$

This and the monotonicity of $u$ imply that for some $C>0$,

$$
0<\lim _{s \rightarrow \infty} u(s)-u(t) \leqslant C \exp (-c t), \quad t \in(0, \infty) .
$$

The proof of (ii) follows from using a rearrangement technique [10]. We can show the monotonicity and the symmetry properties of $u$ for $N \geqslant 2$.

The decay property (iii) $\lim _{|x| \rightarrow \infty} u(t, x)=0$ for $N \geqslant 2$ follows from an elliptic estimate. The decay property (iv) $\lim _{|(t, x)| \rightarrow \infty} u(t, x)=0$ follows from elliptic estimates and the fact that by the Sobolev inequalities for $N \geqslant 3$, $\|u\|_{L^{2 N /(N-2)}}<\infty$. Finally, for $N>3$, we consider a function

$$
\psi_{\beta}(t, x) \equiv \frac{\phi_{\beta, 1}(t)}{|x|^{N-3}},
$$

where $\phi_{\beta, 1}$ is the first eigenfunction of (8) with the corresponding eigenvalue $\lambda_{\beta, 1}$. Then, we see that for $x \neq 0$,

$$
\Delta \psi_{\beta}+\exp (-\beta t) u^{p-1} \psi_{\beta}=\left(u^{p-1}(t, x)-\lambda_{\beta, 1}\right) \exp (-\beta t) \psi_{\beta} .
$$

From above decay property of $u$, we see that for sufficiently large $x \in \mathbf{R}^{N-1}, u^{p-1}(t, x)-\lambda_{\beta, 1}<0$. Thus, by a comparison principle (refer [12]), we see that for some constant $C>0$,

$$
u(t, x) \leqslant C \psi_{\beta}(t, x) \leqslant \frac{C}{|x|^{N-3}} .
$$

This finishes the proof of (v).

Finally, we close up this section with a symmetry property and a non-existence result of positive solutions for Eq. (2) for more general $\Omega$.

Proposition 2.6. For $N \geqslant 2$, let $u \in H(\Omega)$ be a solution of (2) with $\Omega$ replacing $(0, \infty) \times \mathbf{R}^{N-1}$ satisfying

$$
\lim _{|(t, x)| \rightarrow \infty} u(t, x)=0 .
$$

Suppose that for any $(t, x) \in \Omega,(t, y) \in \Omega$ if $|y| \leqslant|x|$. Then, for some $x_{0} \in \mathbf{R}^{N-1}$, $u$ depends only on $t$ and $r \equiv$ $\left|x-x_{0}\right|$, and $\frac{\partial u(t, r)}{\partial r}<0$ for $r \neq 0$ and $t>0$.

Proof. We sketch the proof here since it is standard by now to show the symmetry property of positive solution via a moving plane method [7]. Let $x_{0} \in \mathbf{R}^{N-1} \backslash\{0\}$. For $\lambda>0$, let $T_{\lambda}=\left\{(t, x) \mid\left\langle x_{0}, x\right\rangle=\lambda\right\}$, and $E_{\lambda}=\{(t, x) \in \Omega \mid$ $\left.\left\langle x_{0}, x\right\rangle \geqslant \lambda\right\}$. For $(t, x) \in E_{\lambda}$, we denote $\left(t, x^{\lambda}\right)$ the reflection of $(t, x)$ with respect to $T_{\lambda}$ and define $u_{\lambda}(t, x)=u\left(t, x^{\lambda}\right)$. Define $\bar{\lambda} \equiv \sup \left\{\lambda \in \mathbf{R} \mid E_{\lambda} \neq \emptyset\right\} \in(-\infty, \infty]$

Suppose that for $\lambda$ sufficiently close to $\bar{\lambda}$,

$$
w_{\lambda}(t, x) \equiv \min \left\{u_{\lambda}(t, x)-u(t, x), 0\right\} \neq 0 .
$$


Then, it is easy to deduce that

$$
\int_{E_{\lambda}}\left|\nabla w_{\lambda}\right|^{2} \mathrm{~d} t \mathrm{~d} x \leqslant\left\|p u^{p-1}\right\|_{L^{\infty}\left(E_{\lambda}\right)} \int_{E_{\lambda}} \exp (-\beta t)\left(w_{\lambda}\right)^{2} \mathrm{~d} t \mathrm{~d} x .
$$

Note that for $\bar{\lambda}=\infty, \lim _{\lambda \rightarrow \infty}\left\|p u^{p-1}\right\|_{L^{\infty}\left(E_{\lambda}\right)}=0$, and that for $\bar{\lambda} \neq \infty$, the first eigenvalue $-\Delta$ on $E_{\lambda}$ goes to $\infty$ as $\lambda \rightarrow \bar{\lambda}$. Thus, the inequality (15) contradicts Proposition 2.1. This implies that for $\lambda$ sufficiently close to $\bar{\lambda}, w_{\lambda}>0$ on $E_{\lambda}$. Define $\lambda_{0} \equiv \inf \left\{\lambda \in \mathbf{R} \mid w_{\lambda}>0\right.$ on $\left.E_{\lambda}\right\}>-\infty$. Then, combining above arguments and the Hopf maximum principle, we conclude that $w_{\lambda_{0}} \equiv 0$, that is, $u$ is symmetric for the reflection with respect to $T_{\lambda_{0}}$. Since it holds for any $x_{0} \in \mathbf{R}^{N-1} \backslash\{0\}$, the symmetry and monotonicity properties of $u$ follow.

As in the proof above, for $x_{0} \in \mathbf{R}^{N-1}$ and $\lambda \in \mathbf{R}$, we define

$$
T_{\lambda}=\left\{(t, x) \mid\left\langle x_{0}, x\right\rangle=\lambda\right\} \quad \text { and } \quad E_{\lambda}=\left\{(t, x) \in \Omega \mid\left\langle x_{0}, x\right\rangle \geqslant \lambda\right\} .
$$

For $(t, x) \in E_{\lambda}$, we denote $\left(t, x^{\lambda}\right)$ the reflection of $(t, x)$ with respect to $T_{\lambda}$, and define $E_{\lambda}^{\prime}=\left\{\left(t, x^{\lambda}\right) \mid(t, x) \in E_{\lambda}\right\}$. Therefore, we obtain the following non-existence result.

Proposition 2.7. Suppose that there exists $x_{0} \in \mathbf{R}^{N-1}$ such that for any $\lambda \in \mathbf{R}$ with $E_{\lambda} \neq \emptyset$,

$$
E_{\lambda}^{\prime} \cup E_{\lambda} \subset \Omega \text {, but } E_{\lambda}^{\prime} \cup E_{\lambda} \neq \Omega \text {. }
$$

Then, there exists no solution $u \in H(\Omega)$ for Eq. (2) satisfying $\lim _{|(t, x)| \rightarrow \infty} u(t, x)=0$.

Proof. We sketch the proof here. Denote $\bar{\lambda} \equiv \sup \left\{\lambda \in \mathbf{R} E_{\lambda}^{\prime} \cup E_{\lambda} \subset \Omega \mid E_{\lambda} \neq \emptyset\right\}$ and $\underline{\lambda} \equiv \inf \left\{\lambda \in \mathbf{R} \mid E_{\lambda}^{\prime} \subset \Omega\right\}$. From the fact that $E_{\lambda}^{\prime} \cup E_{\lambda} \neq \Omega$ for any $\lambda \in \mathbf{R}$ with $E_{\lambda} \neq \emptyset$, we see that $\underline{\lambda}=-\infty$.

Suppose that there exists a solution $u \in H(\Omega)$ of (2) satisfying $\lim _{|(t, x)| \rightarrow \infty} u(t, x)=0$. Then, as in the proof of Proposition 2.6, we see that for $\lambda$ sufficiently close to $\bar{\lambda}$,

$$
u\left(t, x^{\lambda}\right)>u(t, x), \quad x \in E_{\lambda} .
$$

Then, since $E_{\lambda}^{\prime} \cup E_{\lambda} \neq \Omega$ for any $\lambda \in \mathbf{R}$ with $E_{\lambda} \neq \emptyset$, by the same argument as in the proof of Proposition 2.6, it follows that for $\lambda<\bar{\lambda}$,

$$
u\left(t, x^{\lambda}\right)>u(t, x), \quad x \in E_{\lambda} .
$$

This contradicts that $\lim _{|(t, x)| \rightarrow \infty} u(t, x)=0$. This proves the claim.

\section{Asymptotic profile of least energy radial solutions on unit ball}

In this section, we consider the limiting behaviour of the least energy radial solutions, i.e., the minimizers of $I^{\mathrm{ral}, \alpha}(B(0,1))$. We consider both the asymptotics of limiting energy and limiting profile.

Let $\Omega=B(0,1) \equiv\left\{x \in \mathbf{R}^{N}|| x \mid<1\right\}$, and $H_{\text {rad }} \equiv\left\{u \in H_{0}^{1,2}(\Omega) \mid u(x)=u(|x|)\right\}$. Then, we consider the following minimization problem

$$
I^{\mathrm{rad}, \alpha} \equiv \inf \left\{\left.\int_{\Omega}|\nabla u|^{2} \mathrm{~d} x\left|\int_{\Omega}\right| x\right|^{\alpha} u^{p+1} \mathrm{~d} x=1, u \in H_{\mathrm{rad}}\right\} .
$$

In [11], Ni proved that the above minimization problem has a positive minimizer $u_{\mathrm{rad}}^{\alpha}$ for $1 \leqslant p<(N+2+2 \alpha) /$ $(N-2)$. Moreover, from the Pohozaev identity, we can show that there is no solution of Eq. (1) with $\Omega=B(0,1)$ for $p \geqslant(N+2+2 \alpha) /(N-2)$. This $u_{\alpha}^{\mathrm{rad}}$ satisfies the following equation

$$
\frac{\partial^{2} u}{\partial r^{2}}+\frac{N-1}{r} \frac{\partial u}{\partial r}+I^{\mathrm{rad}, \alpha}|x|^{\alpha} u^{p}=0 \quad \text { in } \Omega, \quad u=0 \quad \text { on } \partial \Omega .
$$

In [14], it was shown that for $N \geqslant 2, \lim _{\alpha \rightarrow \infty}\left(\frac{N}{\alpha+N}\right)^{\frac{p+3}{p+1}} I^{\mathrm{rad}, \alpha} \in(0, \infty)$. We will examine the exact value of the limit $I^{\mathrm{rad}, \alpha}$ and our analysis applies to $N=1$ too. This analysis in turn will be used to find a fine asymptotic behaviour of the minimizers $u_{\alpha}^{\mathrm{rad}}$ for $I^{\mathrm{rad}, \alpha}$ as $\alpha \rightarrow \infty$. We have the following asymptotic result for $I^{\mathrm{rad}, \alpha}$ and $u_{\alpha}^{\mathrm{rad}}$. We denote $J_{N, \beta} \equiv J_{N, \beta}\left((0, \infty) \times \mathbf{R}^{N-1}\right)$. 
Theorem 3.1. Let $N \geqslant 1$ and $p \geqslant 1$. Then

$$
\lim _{\alpha \rightarrow \infty}\left(\frac{N}{\alpha+N}\right)^{\frac{p+3}{p+1}} I^{\mathrm{rad}, \alpha}=\left|S^{N-1}\right|^{(p-1) /(p+1)} J_{1, N},
$$

where $\left|S^{N-1}\right|$ is the volume of $(N-1)$-dimensional sphere $S^{N-1}$. For any $r \in(0,1], \mathrm{d} u_{\alpha}^{\mathrm{rad}}(r) / \mathrm{d} r<0$. Furthermore, under the following transformation

$$
v_{\alpha}^{\mathrm{rad}}(t) \equiv\left|S^{N-1}\right|^{\frac{1}{p+1}}\left(\frac{N}{\alpha+N}\right)^{\frac{1}{p+1}} u_{\alpha}^{\mathrm{rad}}\left(\exp \left(-\frac{N}{\alpha+N} t\right)\right)
$$

$v_{\alpha}^{\mathrm{rad}}(t)$ converges uniformly on $(0, \infty)$ to a minimizer of $J_{1, N}$ as $\alpha \rightarrow \infty$.

For a nonnegative minimizer $u_{\alpha}^{\mathrm{rad}}$ of $I_{\alpha}^{\mathrm{rad}}$, a scaled function $\omega_{\alpha}^{\mathrm{rad}} \equiv\left(I_{\alpha}^{\mathrm{rad}}\right)^{\frac{1}{p-1}} u_{\alpha}^{\mathrm{rad}}$ is a least energy solution (a mountain pass solution) of (1) in the class of radial functions $H_{\text {rad }}$. Proposition 2.3 implies that there exists a least energy solution (a mountain pass solution) of $\omega_{N, \beta}$ in $H\left((0, \infty) \times \mathbf{R}^{N-1}\right)$ of the equation

$$
\begin{aligned}
& \Delta u+\exp (-\beta t) u^{p}=0 \quad \text { in }(0, \infty) \times \mathbf{R}^{N-1}, \\
& u>0 \text { in }(0, \infty) \times \mathbf{R}^{N-1}, \\
& u=0 \quad \text { on }\{0\} \times \mathbf{R}^{N-1} .
\end{aligned}
$$

For the minimizer $u_{N, \beta}$ of $J_{N, \beta} \equiv J_{N, \beta}\left((0, \infty) \times \mathbf{R}^{N-1}\right)$, the least energy solution $\omega_{N, \beta}$ of (17) is given by $\left(J_{N, \beta}\right)^{\frac{1}{p-1}} u_{N, \beta}$. Then, we have the following equivalent version of Theorem 3.1 for $\omega_{\alpha}^{\text {rad }}$ and its energy.

Theorem 3.1-E. Let $N \geqslant 1$ and $p \geqslant 1$. Then

$$
\begin{gathered}
\lim _{\alpha \rightarrow \infty}\left(\frac{N}{\alpha+N}\right)^{\frac{p+3}{p-1}} \int_{B(0,1)} \frac{1}{2}\left|\nabla \omega_{\alpha}^{\mathrm{rad}}\right|^{2}-\frac{1}{p+1}|x|^{\alpha}\left(\omega_{\alpha}^{\mathrm{rad}}\right)^{p+1} \mathrm{~d} x \\
=\left|S^{N-1}\right| \int_{0}^{\infty} \frac{1}{2}\left|\nabla \omega_{1, N}\right|^{2}-\frac{1}{p+1} \exp (-N t)\left(\omega_{1, N}\right)^{p+1} \mathrm{~d} t,
\end{gathered}
$$

where $\left|S^{N-1}\right|$ is the volume of $(N-1)$-dimensional sphere $S^{N-1}$. Furthermore, the following transformed solution

$$
W_{\alpha}^{\mathrm{rad}}(t) \equiv\left|S^{N-1}\right|^{p}\left(\frac{N}{\alpha+N}\right)^{\frac{2}{p-1}} \omega_{\alpha}^{\mathrm{rad}}\left(\exp \left(-\frac{N}{\alpha+N} t\right)\right)
$$

converges uniformly on $(0, \infty)$ to a least energy solution $\omega_{1, N}$ of (17) as $\alpha \rightarrow \infty$.

Proof of Theorem 3.1. It is easy to see that there are no local minimum points of $u_{\alpha}^{\mathrm{rad}}$ in $(0,1)$. Suppose that there exists $r_{0} \in(0,1)$ satisfying $u_{\alpha}^{\mathrm{rad}}(0)<u_{\alpha}^{\mathrm{rad}}(r)$ for any $r \in\left(0, r_{0}\right)$. Then, defining $w_{\alpha}^{\mathrm{rad}}=u_{\alpha}^{\mathrm{rad}}(r)$ for $r \in\left(r_{0}, 1\right)$ and $u_{\alpha}^{\mathrm{rad}}\left(r_{0}\right)$ for $r \in\left[0, r_{0}\right]$, we see that

$$
\int_{\Omega}\left|\nabla u_{\alpha}^{\mathrm{rad}}\right|^{2} \mathrm{~d} x>\int_{\Omega}\left|\nabla w_{\alpha}^{\mathrm{rad}}\right|^{2} \mathrm{~d} x
$$

and

$$
\int_{\Omega}|x|^{\alpha}\left(u_{\alpha}^{\mathrm{rad}}\right)^{p+1} \mathrm{~d} x<\int_{\Omega}|x|^{\alpha}\left(w_{\alpha}^{\mathrm{rad}}\right)^{p+1} \mathrm{~d} x .
$$

This implies that

$$
\frac{\int_{\Omega}\left|\nabla w_{\alpha}^{\mathrm{rad}}\right|^{2} \mathrm{~d} x}{\left(\int_{\Omega}|x|^{\alpha}\left(w_{\alpha}^{\mathrm{rad}}\right)^{p+1} \mathrm{~d} x\right)^{2 /(p+1)}}<\frac{\int_{\Omega}\left|\nabla u_{\alpha}^{\mathrm{rad}}\right|^{2} \mathrm{~d} x}{\left(\int_{\Omega}|x|^{\alpha}\left(u_{\alpha}^{\mathrm{rad}}\right)^{p+1} \mathrm{~d} x\right)^{2 /(p+1)}} ;
$$


this contradicts that $u_{\alpha}^{\mathrm{rad}}$ is a minimizer of $I^{\mathrm{rad}, \alpha}$. Thus we see that $u_{\mathrm{rad}}^{\alpha}$ is monotone decreasing on $[0,1]$.

We transform $u_{\alpha}^{\mathrm{rad}}$ as follows: for $t \in(0, \infty)$,

$$
v_{\alpha}^{\mathrm{rad}}(t) \equiv\left|S^{N-1}\right|^{\frac{1}{p+1}}\left(\frac{N}{\alpha+N}\right)^{\frac{1}{p+1}} u_{\alpha}^{\mathrm{rad}}\left(\exp \left(-\frac{N}{\alpha+N} t\right)\right) .
$$

Then, direct calculations show

$$
\int_{\Omega}\left|\nabla u_{\alpha}^{\mathrm{rad}}\right|^{2} \mathrm{~d} x=\left|S^{N-1}\right|^{\frac{p-1}{p+1}}\left(\frac{\alpha+N}{N}\right)^{\frac{p+3}{p+1}} \int_{0}^{\infty} \exp \left(-\frac{N(N-2) t}{\alpha+N}\right)\left|\frac{\mathrm{d} v_{\alpha}^{\mathrm{rad}}}{\mathrm{d} t}\right|^{2} \mathrm{~d} t,
$$

and

$$
\int_{\Omega}|x|^{\alpha}\left(u_{\alpha}^{\mathrm{rad}}\right)^{p+1} \mathrm{~d} x=\int_{0}^{\infty} \exp (-N t)\left(v_{\alpha}^{\mathrm{rad}}\right)^{p+1} \mathrm{~d} t .
$$

Thus, we see that for any $p \geqslant 1$,

$$
\left(\frac{N}{\alpha+N}\right)^{\frac{p+3}{p+1}} I^{\mathrm{rad}, \alpha}=\left|S^{N-1}\right|^{\frac{p-1}{p+1}} \int_{0}^{\infty} \exp \left(-\frac{N(N-2) t}{\alpha+N}\right)\left|\frac{\mathrm{d} v_{\alpha}^{\mathrm{rad}}}{\mathrm{d} t}\right|^{2} \mathrm{~d} t
$$

and

$$
\int_{0}^{\infty} \exp (-N t)\left(v_{\alpha}^{\mathrm{rad}}\right)^{p+1} \mathrm{~d} t=1
$$

Moreover, it follows that

$$
\begin{aligned}
& \frac{\mathrm{d}}{\mathrm{d} t}\left(\exp \left(-\frac{N(N-2) t}{\alpha+N}\right) \frac{\mathrm{d} v_{\alpha}^{\mathrm{rad}}}{\mathrm{d} t}\right)+H_{\alpha} \exp (-N t)\left(v_{\alpha}^{\mathrm{rad}}\right)^{p}=0 \quad \text { on }(0, \infty), \\
& \lim _{t \rightarrow \infty} \frac{\mathrm{d} v_{\alpha}^{\mathrm{rad}}(t)}{\mathrm{d} t}=v_{\alpha}^{\mathrm{rad}}(0)=0,
\end{aligned}
$$

where

$$
H_{\alpha} \equiv\left|S^{N-1}\right|^{-\frac{p-1}{p+1}}\left(\frac{N}{\alpha+N}\right)^{\frac{p+3}{p+1}} I^{\mathrm{rad}, \alpha} .
$$

Since $\lim _{\alpha \rightarrow \infty} \exp \left(-\frac{N(N-2) t}{\alpha+N}\right)=1$ uniformly on each compact subset of $[0, \infty)$, it follows that

$$
\lim _{\alpha \rightarrow \infty}\left(\frac{N}{\alpha+N}\right)^{\frac{p+3}{p+1}} I^{\mathrm{rad}, \alpha} \leqslant\left|S^{N-1}\right|^{(p-1) /(p+1)} J_{1, N} .
$$

This implies that $\lim _{\sup } \operatorname{su\infty }_{\alpha \rightarrow \infty} H_{\alpha} \leqslant J_{1, N}$.

For sufficiently large $\alpha>0$, we see that

$$
\begin{aligned}
\int_{0}^{\infty} \exp (-N t)\left|\frac{\mathrm{d} v_{\alpha}^{\mathrm{rad}}}{\mathrm{d} t}\right|^{2} \mathrm{~d} t & \leqslant \int_{0}^{\infty} \exp \left(-\frac{N(N-2) t}{\alpha+N}\right)\left|\frac{\mathrm{d} v_{\alpha}^{\mathrm{rad}}}{\mathrm{d} t}\right|^{2} \mathrm{~d} t \\
& =H_{\alpha} \int_{0}^{\infty} \exp (-N t)\left(v_{\alpha}^{\mathrm{rad}}\right)^{p+1} \mathrm{~d} t \leqslant H_{\alpha}\left\|v_{\alpha}^{\mathrm{rad}}\right\|_{L^{\infty}}^{p-1} \int_{0}^{\infty} \exp (-N t)\left(v_{\alpha}^{\mathrm{rad}}\right)^{2} \mathrm{~d} t
\end{aligned}
$$

Then, from the inequality (4), we see that $\left\{\left\|v_{\alpha}^{\mathrm{rad}}\right\|_{L^{\infty}}\right\}_{\alpha}$ is bounded away from 0 . 
From now, we will show that $\left\{\left\|v_{\alpha}^{\mathrm{rad}}\right\|_{L^{\infty}}\right\}_{\alpha}$ is bounded. Defining $W_{\alpha} \equiv \mathrm{d} v_{\alpha}^{\mathrm{rad}} / \mathrm{d} t$, we see from the equation for $v_{\alpha}^{\mathrm{rad}}$ that

$$
\frac{\mathrm{d}^{2} W_{\alpha}}{\mathrm{d} t^{2}}+\frac{N(\alpha-N+4)}{\alpha+N} \frac{\mathrm{d} W_{\alpha}}{\mathrm{d} t}+\left(p H_{\alpha} \exp \left(-\frac{N(\alpha+2)}{\alpha+N} t\right)\left(v_{\alpha}^{\mathrm{rad}}\right)^{p-1}-\frac{N^{2}(\alpha+2)(N-2)}{(\alpha+N)^{2}}\right) W_{\alpha}=0 .
$$

Note that $v_{\alpha}^{\mathrm{rad}}(0)=0, \lim _{t \rightarrow \infty} W_{\alpha}(t)=0$. Then, we see that

$$
v_{\alpha}^{\mathrm{rad}}(t)=\int_{0}^{t} W_{\alpha}(s) \mathrm{d} s=\int_{0}^{t} \exp \left(\frac{N(N-2)}{2(\alpha+N)} s\right) \exp \left(-\frac{N(N-2)}{2(\alpha+N)} s\right) W_{\alpha} \mathrm{d} s .
$$

Then for some constant $C>0$, independent of $\alpha$,

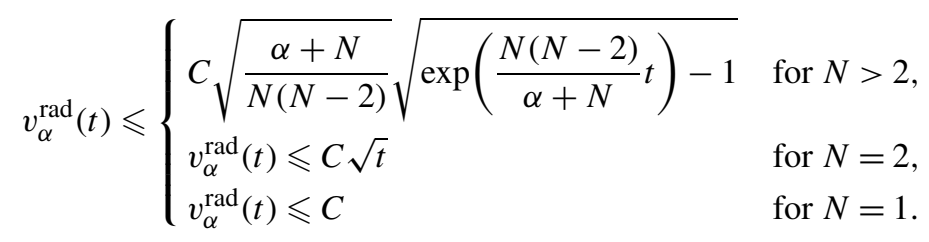

Note that for any $\gamma, c, t>0$,

$$
\exp (-c t) \gamma\left(\exp \left(\frac{t}{\gamma}\right)-1\right) \leqslant t \exp \left(\left(\frac{1}{\gamma}-c\right) t\right)
$$

Thus we see from (18) that

$$
\lim _{|(\alpha, t)| \rightarrow \infty}\left(p H_{\alpha} \exp \left(-\frac{N(\alpha+2)}{\alpha+N} t\right)\left(v_{\alpha}^{\mathrm{rad}}\right)^{p-1}-\frac{N^{2}(\alpha+2)(N-2)}{(\alpha+N)^{2}}\right)=0 .
$$

It is standard to show that for each $T>0,\left\{\left\|W_{\alpha}\right\|_{L^{\infty}(0, T)}\right\}_{\alpha}$ is bounded. For $a \in(0, N)$, we denote $\phi(t) \equiv \exp (-a t)$, Then, we see that for sufficiently large $\alpha>0$ and $t>0$,

$$
\frac{\mathrm{d}^{2} \phi}{\mathrm{d} t^{2}}+\frac{N(\alpha-N+4)}{\alpha+N} \frac{\mathrm{d} \phi}{\mathrm{d} t}+\left(p H_{\alpha} \exp \left(-\frac{N(\alpha+2)}{\alpha+N} t\right)\left(v_{\alpha}^{\mathrm{rad}}\right)^{p-1}-\frac{N^{2}(\alpha+2)(N-2)}{(\alpha+N)^{2}}\right) \phi \leqslant 0 .
$$

Then it follows from the comparison principle that for any given $a \in(0, N)$, there exists some $C>0$, independent of $\alpha>0$, satisfying

$$
W_{\alpha}(t) \leqslant C \exp (-a t), \quad t \geqslant 0 .
$$

Then, since $v_{\alpha}^{\mathrm{rad}}(t)=\int_{0}^{t} W_{\alpha}(s) \mathrm{d} s$, it follows that $\left\{\left\|v_{\alpha}^{\mathrm{rad}}\right\|_{L^{\infty}}\right\}_{\alpha}$ is bounded, and that for any $a \in(0, N)$, there exists some $C>0$ satisfying

$$
\lim _{s \rightarrow \infty} v_{\alpha}^{\mathrm{rad}}(s)-v_{\alpha}^{\mathrm{rad}}(t) \leqslant C \exp (-a t), \quad t>0 .
$$

Now, from the elliptic estimates [8], we deduce that for each $T>0$ and $\gamma \in(0,1),\left\{\left|v_{\alpha}^{\mathrm{rad}}\right|_{C^{2, \gamma}(0, T)}\right\}_{\alpha}$ is bounded. If $\liminf _{\alpha \rightarrow \infty}\left\|v_{\alpha}^{\operatorname{rad}}\right\|_{L^{\infty}((0, T))}=0$ for sufficiently large $T>0$, from the boundedness of $\left\{\left\|v_{\alpha}^{\operatorname{rad}}\right\|_{L^{\infty}}\right\}_{\alpha}$, it follows that

$$
\liminf _{\alpha \rightarrow \infty} \int_{0}^{\infty} \exp (-N t)\left(v_{\alpha}^{\mathrm{rad}}\right)^{p+1} \mathrm{~d} t=0 ;
$$

this contradicts that for any $\alpha>0, \int_{0}^{\infty} \exp (-N t)\left(v_{\alpha}^{\mathrm{rad}}\right)^{p+1} \mathrm{~d} t=1$. Thus, we deduce that for some $H \in\left(0, J_{1, N}\right]$, the solution $v_{\alpha}^{\text {rad }}$ converges in $C_{\text {loc }}^{2}(0, \infty)$ to a solution $v \in H((0, \infty))$ of

$$
\begin{aligned}
& \frac{\mathrm{d}^{2} v}{\mathrm{~d} t^{2}}+H \exp (-N t) v^{p}=0, \quad v>0 \quad \text { in }(0, \infty), \\
& v(0)=0 .
\end{aligned}
$$


Since $\int_{0}^{\infty} \exp (-N t) v^{p+1} \mathrm{~d} t=1$, it follows that $H=\int_{0}^{\infty}\left|\frac{\mathrm{d} v}{\mathrm{~d} t}\right|^{2} \mathrm{~d} t \geqslant J_{1, N}$. Therefore, it follows that

$$
\lim _{\alpha \rightarrow \infty}\left(\frac{N}{\alpha+N}\right)^{\frac{p+3}{p+1}} I^{\mathrm{rad}, \alpha} \geqslant\left|S^{N-1}\right|^{\frac{p-1}{p+1}} J_{1, N} .
$$

Thus we get

$$
\lim _{\alpha \rightarrow \infty}\left(\frac{N}{\alpha+N}\right)^{\frac{p+3}{p+1}} I^{\mathrm{rad}, \alpha}=\left|S^{N-1}\right|^{\frac{p-1}{p+1}} J_{1, N} .
$$

Moreover, from (19), it follows that $v_{\alpha}^{\text {rad }}$ converges uniformly to a minimizer of $J_{1, N}$. This completes the proof.

\section{Asymptotic profile of least energy solutions on the unit ball}

In this section, we turn to the least energy solutions of the Hénon equation (1). We will study both asymptotic energy and asymptotic profile of the ground states.

Let us consider the following minimization problem

$$
I^{\mathrm{all}, \alpha} \equiv \inf \left\{\left.\|u\|^{2}\left|\int_{\Omega}\right| x\right|^{\alpha} u^{p+1} \mathrm{~d} x=1, u \in H_{0}^{1,2}(B(0,1))\right\} .
$$

For $p \in[1,(N+2) /(N-2))$, there exists a positive minimizer $u_{\alpha}^{\text {all }}$ of $(21)$. This $u_{\alpha}^{\text {all }}$ satisfies the following equation

$$
\Delta u+I^{\text {all }, \alpha}|x|^{\alpha} u^{p}=0, \quad u>0 \text { in } \Omega, \quad u=0 \quad \text { on } \partial \Omega .
$$

When $N \geqslant 2$, for $x \in \mathbf{R}^{N}$, we take polar coordinates $x=(r, \theta)$ with $r=|x| \in[0, \infty), \theta=x /|x| \in S^{N-1}$, and denote $u(x)=u(r, \theta)$. For the sake of convenience, we denote $S_{\alpha}^{N-1} \equiv \frac{\alpha+N}{N} S^{N-1}$. For each $y \in \mathbf{R}^{N-1} \equiv \mathbf{R}^{N-1} \times\{0\} \subset \mathbf{R}^{N}$, there exists a unique $\psi_{\alpha}(y) \in S_{\alpha}^{N-1} \backslash\left\{\left(0, \ldots, 0, \frac{\alpha+N}{N}\right)\right\}$ such that $t(y) \psi_{\alpha}(y)+(1-t(y))\left(0, \ldots, 0, \frac{\alpha+N}{N}\right)=y$ for some $t(y)>0$ depending upon $\alpha$. Then, the map $\left(\psi_{\alpha}\right)^{-1}: S_{\alpha}^{N-1} \backslash\left\{\left(0, \ldots, 0, \frac{\alpha+N}{N}\right)\right\} \rightarrow \mathbf{R}^{N-1}$ is a stereographic projection. Also when $N \geqslant 2$, by a rearrangement technique [10], we can assume that $u_{\alpha}^{\text {all }}(x)=u_{\alpha}^{\text {all }}(g \cdot x)$ for $g \in O(N-1) \otimes I \subset$ $O(N)$ (i.e., $u$ is radially symmetric with respect to the first $N-1$ variables), and that for fixed $r \in(0,1), u_{\alpha}^{\text {all }}(r, \theta)$ decreases strictly as $|\theta-(0, \ldots, 0,-1)|$ increases. If $N=1$, we can assume that $\mathrm{d} u_{\alpha}^{\text {all }}(0) / \mathrm{d} x \geqslant 0$.

Then, we have the following results on the asymptotic behaviour of the least energy $I_{\alpha}^{\text {all }}$ and the minimizer $u_{\alpha}^{\text {all }}$.

Theorem 4.1. Let $p \in\left(1,2^{*}-1\right)$. Then

$$
\lim _{\alpha \rightarrow \infty}\left(\frac{N}{\alpha+N}\right)^{\frac{N+2-(N-2) p}{p+1}} I^{\text {all }, \alpha}=J_{N, N} .
$$

Moreover, the following transformed solution

$$
V_{\alpha}^{\text {all }}(t, y) \equiv \begin{cases}\left(\frac{N}{\alpha+N}\right)^{\frac{N}{p+1}} u_{\alpha}^{\text {all }}\left(\exp \left(-\frac{N}{\alpha+N} t\right), \frac{N}{\alpha+N} \psi_{\alpha}(y)\right) & \text { for } N \geqslant 3, \\ \left(\frac{2}{\alpha+2}\right)^{\frac{2}{p+1}} u_{\alpha}^{\text {all }}\left(\exp \left(-\frac{2}{\alpha+2} t\right), \frac{2}{\alpha+2} y\right) & \text { for } N=2, \\ \left(\frac{1}{\alpha+1}\right)^{\frac{1}{p+1}} u_{\alpha}^{\text {all }}\left(\exp \left(-\frac{t}{\alpha+1}\right)\right) & \text { for } N=1\end{cases}
$$

with $t \in[0, \infty), y \in \mathbf{R}^{N-1}$, converges to a minimizer of $J_{N, N}$ uniformly for $N \geqslant 3$ and locally uniformly for $N=1,2$ as $\alpha \rightarrow \infty$, and, for $N=1$, the following transformed solution $\left(\frac{1}{\alpha+1}\right)^{1 /(p+1)} u_{\alpha}^{\text {all }}\left(-\exp \left(-\frac{t}{\alpha+1}\right)\right.$ ) converges locally uniformly to 0 as $\alpha \rightarrow \infty$.

For a nonnegative minimizer $u_{\alpha}^{\text {all }}$ of $I_{\alpha}^{\text {all }}$, a scaled function $\omega_{\alpha}^{\text {all }} \equiv\left(I_{\alpha}^{\text {all }}\right)^{\frac{1}{p-1}} u_{\alpha}^{\text {all }}$ is a least energy solution (a mountain pass solution) of (1) in the whole class of functions in $H_{0}^{1,2}(B(0,1))$. Then, we have the following equivalent version of Theorem 4.1 as for $\omega_{\alpha}^{\mathrm{rad}}$ and its energy in Theorem 3.1-E. 
Theorem 4.1-E. Let $p \in\left(1,2^{*}-1\right)$. Then

$$
\begin{aligned}
& \lim _{\alpha \rightarrow \infty}\left(\frac{N}{\alpha+N}\right)^{\frac{N+2-(N-2) p}{p-1}} \int_{B(0,1)} \frac{1}{2}\left|\nabla \omega_{\alpha}^{\mathrm{all}}\right|^{2}-\frac{1}{p+1}|x|^{\alpha}\left(\omega_{\alpha}^{\mathrm{all}}\right)^{p+1} \mathrm{~d} x \\
& =\int_{0}^{\infty} \frac{1}{2}\left|\nabla \omega_{N, N}\right|^{2}-\frac{1}{p+1} \exp (-N t)\left(\omega_{N, N}\right)^{p+1} \mathrm{~d} t
\end{aligned}
$$

for some $\omega_{N, N}$ being a least energy solution of $(14-(\mathrm{N}, \mathrm{N}))$. Moreover, the following transformed solution

$$
W_{\alpha}^{\text {all }}(t, y) \equiv \begin{cases}\left(\frac{N}{\alpha+N}\right)^{\frac{2}{p-1}} \omega_{\alpha}^{\text {all }}\left(\exp \left(-\frac{N}{\alpha+N} t\right), \frac{N}{\alpha+N} \psi_{\alpha}(y)\right) & \text { for } N \geqslant 3, \\ \left(\frac{2}{\alpha+2}\right)^{\frac{2}{p-1}} \omega_{\alpha}^{\text {all }}\left(\exp \left(-\frac{2}{\alpha+2} t\right), \frac{2}{\alpha+2} y\right) & \text { for } N=2, \\ \left(\frac{1}{\alpha+1}\right)^{\frac{2}{p-1}} \omega_{\alpha}^{\text {all }}\left(\exp \left(-\frac{t}{\alpha+1}\right)\right) & \text { for } N=1\end{cases}
$$

with $t \in[0, \infty), y \in \mathbf{R}^{N-1}$, converges to $\omega_{N, N}$ uniformly for $N \geqslant 3$ and locally uniformly for $N=1,2$ as $\alpha \rightarrow \infty$, and, for $N=1$, the following transformed solution $\left(\frac{1}{\alpha+1}\right)^{\frac{2}{p-1}} \omega_{\alpha}^{\text {all }}\left(-\exp \left(-\frac{t}{\alpha+1}\right)\right)$ converges locally uniformly to 0 as $\alpha \rightarrow \infty$.

Proof of Theorem 4.1. We take polar coordinates $x=(r, \theta)$ with $r \in[0, \infty), \theta \in S^{N-1}$, and denote $u(x)=u(r, \theta)$. We first consider the following transformation

$$
v_{\alpha}^{\text {all }}(t, \phi) \equiv\left(\frac{\alpha+N}{N}\right)^{-\frac{N}{p+1}} u_{\alpha}^{\text {all }}\left(\exp \left(-\frac{N}{\alpha+N} t\right), \frac{N}{\alpha+N} \phi\right),
$$

where $t \in[0, \infty)$ and $\phi \in S_{\alpha}^{N-1}$. For the sake of convenience, we denote $\mathrm{d}_{\alpha} \sigma$ the volume element of $S_{\alpha}^{N-1}$. Then, from some direct calculations, we get for $N \geqslant 2$

$$
\begin{aligned}
& I^{\mathrm{all}, \alpha}=\int_{\Omega}\left|\nabla u_{\alpha}^{\mathrm{all}}\right|^{2} \mathrm{~d} x=\left(\frac{\alpha+N}{N}\right)^{\frac{N+2-(N-2) p}{p+1}} \int_{(0, \infty) \times S_{\alpha}^{N-1}} \exp \left(-\frac{N(N-2) t}{\alpha+N}\right)\left(\left|\frac{\partial v_{\alpha}^{\text {all }}}{\partial t}\right|^{2}+\left|\nabla_{S_{\alpha}} v_{\alpha}^{\mathrm{all}}\right|^{2}\right) \mathrm{d} t \mathrm{~d}_{\alpha} \sigma, \\
& 1=\int_{\Omega}|x|^{\alpha}\left(u_{\alpha}^{\mathrm{all}}\right)^{p+1} \mathrm{~d} x=\int_{(0, \infty) \times S_{\alpha}^{N-1}} \exp (-N t)\left(v_{\alpha}^{\mathrm{all}}\right)^{p+1} \mathrm{~d} t \mathrm{~d}_{\alpha} \sigma,
\end{aligned}
$$

where $\nabla_{S_{\alpha}}$ is the gradient on $S_{\alpha}^{N-1}$. Thus we see that $K_{\alpha, N} \equiv I^{\text {all }, \alpha}\left(\frac{N}{\alpha+N}\right)^{\frac{N+2-(N-2) p}{p+1}}$,

$$
\frac{\partial^{2} v_{\alpha}^{\text {all }}}{\partial t^{2}}-\frac{N(N-2)}{\alpha+N} \frac{\partial v_{\alpha}^{\text {all }}}{\partial t}+\Delta_{S_{\alpha}^{N-1}} v_{\alpha}^{\text {all }}+K_{\alpha, N} \exp \left(-\frac{N(\alpha+2) t}{\alpha+N}\right)\left(v_{\alpha}^{\text {all }}\right)^{p}=0
$$

in $(0, \infty) \times S_{\alpha}$ and $v_{\alpha}^{\text {all }}=0$ on $\{0\} \times S_{\alpha}^{N-1}$. A direct computation shows that (22) also holds for $N=1$.

For each $\varphi \in C_{0}^{\infty}\left((0, \infty) \times \mathbf{R}^{N-1}\right)$, we define a function $w_{\alpha} \in C_{0}^{\infty}(B(0,1))$

$$
w_{\alpha}(r, \theta) \equiv\left\{\begin{array}{l}
\left(\frac{\alpha+N}{N}\right)^{\frac{N}{p+1}} \varphi\left(-\frac{\alpha+N}{N} \log r,\left(\psi_{\alpha}\right)^{-1}\left(\frac{\alpha+N}{N} \theta\right)\right) \text { for } N \geqslant 2, \\
\left(\frac{\alpha+N}{N}\right)^{\frac{N}{p+1}} \varphi\left(-\frac{\alpha+N}{N} \log r\right) \quad \text { for } N=1 .
\end{array}\right.
$$

Then, since $\varphi \in C_{0}^{\infty}\left((0, \infty) \times \mathbf{R}^{N-1}\right)$, it is not difficult to deduce that 


$$
\begin{aligned}
& \frac{\int_{\Omega}\left|\nabla w_{\alpha}\right|^{2} \mathrm{~d} x}{\left(\int_{\Omega}|x|^{\alpha}\left|w_{\alpha}\right|^{p+1} \mathrm{~d} x\right)^{2 /(p+1)}} \\
& =\left(\frac{\alpha+N}{N}\right)^{\frac{N+2-(N-2) p}{p+1}} \frac{\int_{(0, \infty) \times \mathbf{R}^{N-1}} \exp \left(-\frac{N(N-2) t}{\alpha+N}\right)\left(\left|\frac{\partial \varphi}{\partial t}\right|^{2}+\left|\nabla_{y} \varphi\right|^{2}\right) \mathrm{d} t \mathrm{~d} y+\mathrm{O}\left(\frac{1}{\alpha}\right)}{\left(\int_{(0, \infty) \times \mathbf{R}^{N-1}} \exp (-N t)|\varphi|^{p+1} \mathrm{~d} t \mathrm{~d} y+\mathrm{O}\left(\frac{1}{\alpha}\right)\right)^{2 /(p+1)}}
\end{aligned}
$$

as $\alpha \rightarrow \infty$. Furthermore, since $\varphi \in C_{0}^{\infty}\left((0, \infty) \times \mathbf{R}^{N-1}\right)$, it follows that

$$
\begin{gathered}
\lim _{\alpha \rightarrow \infty} \frac{\int_{(0, \infty) \times \mathbf{R}^{N-1}} \exp \left(-\frac{N(N-2) t}{\alpha+N}\right)\left|\frac{\partial \varphi}{\partial t}\right|^{2}+\left|\nabla_{y} \varphi\right|^{2} \mathrm{~d} t \mathrm{~d} y+\mathrm{O}\left(\frac{1}{\alpha}\right)}{\left(\int_{(0, \infty) \times \mathbf{R}^{N-1}} \exp (-N t)|\varphi|^{p+1} \mathrm{~d} t \mathrm{~d} y+\mathrm{O}\left(\frac{1}{\alpha}\right)\right)^{2 /(p+1)}} \\
=\frac{\int_{(0, \infty) \times \mathbf{R}^{N-1}|\nabla \varphi|^{2} \mathrm{~d} t \mathrm{~d} y}}{\left(\int_{(0, \infty) \times \mathbf{R}^{N-1}} \exp (-N t)|\varphi|^{p+1} \mathrm{~d} t \mathrm{~d} y\right)^{2 /(p+1)}} .
\end{gathered}
$$

This implies that

$$
\lim _{\alpha \rightarrow \infty}\left(\frac{N}{\alpha+N}\right)^{\frac{N+2-(N-2) p}{p+1}} I^{\text {all }, \alpha} \leqslant J_{N, N} .
$$

Next, by a similar argument as in the proof of Theorem 3.1, we deduce using (5) that for $N \geqslant 2,\left\{\left\|v_{\alpha}^{\text {all }}\right\|_{L^{\infty}}\right\}_{\alpha}$ is bounded away from 0 . For $N=1$, since $v_{\alpha}^{\text {all }}(t)=(\alpha+1)^{\frac{-1}{p+1}} u_{\alpha}^{\text {all }}\left(\exp \left(\frac{-t}{\alpha+1}\right)\right)$, we see that

$$
\frac{\mathrm{d}}{\mathrm{d} t}\left(\exp \left(\frac{t}{\alpha+1}\right) \frac{\mathrm{d} v_{\alpha}^{\mathrm{all}}}{\mathrm{d} t}\right)+I^{\mathrm{all}, \alpha}(\alpha+1)^{-\frac{p+3}{p+1}} \exp (-t)\left(v_{\alpha}^{\text {all }}\right)^{p}=0 \quad \text { on }(0, \infty),
$$

and that

$$
v_{\alpha}^{\text {all }}(0)=0, \quad \lim _{t \rightarrow \infty} \exp \left(\frac{t}{\alpha+1}\right) \frac{\mathrm{d} v_{\alpha}^{\text {all }}(t)}{\mathrm{d} t}=-\frac{\mathrm{d} u_{\alpha}^{\text {all }}(0)}{\mathrm{d} x} \frac{1}{(\alpha+1)^{\frac{p+2}{p+1}}} .
$$

We assumed that $\frac{\mathrm{d} u_{\alpha}^{\text {all }}}{\mathrm{d} x}(0) \geqslant 0$. Then, since $\frac{\mathrm{d}^{2} u_{\alpha}^{\text {all }}}{\mathrm{d} x^{2}}(x) \leqslant 0$ for $|x|<1$, the unique maximum point of $u_{\alpha}^{\text {all }}$ is located in $[0,1)$. From Theorem 3.1 and (23), we see that $u_{\alpha}^{\text {all }}$ is not symmetric, that is, $u_{\alpha}^{\text {all }}(x) \neq u_{\alpha}^{\text {all }}(-x)$ for some $x \in(0,1)$. Thus, from the uniqueness of a solution for the initial value problem of ordinary differential equations, we deduce that $\frac{\mathrm{d} u_{\alpha}^{\text {all }}}{\mathrm{d} x}(0) \neq 0$; then $\frac{\mathrm{d} u_{\alpha}^{\text {all }}}{\mathrm{d} x}(0)>0$. Multiplying $v_{\alpha}^{\text {all }}$ on both sides of $(24)$ and integrating by parts, we get

$$
\begin{aligned}
& \int_{0}^{\infty} \exp \left(\frac{t}{\alpha+1}\right)\left|\frac{\mathrm{d} v_{\alpha}^{\text {all }}}{\mathrm{d} t}\right|^{2} \mathrm{~d} t-\lim _{t \rightarrow \infty} \exp \left(\frac{t}{\alpha+1}\right) v_{\alpha}^{\text {all }}(t) \frac{\mathrm{d} v_{\alpha}^{\text {all }}(t)}{\mathrm{d} t} \\
& =\int_{0}^{\infty} \exp \left(\frac{t}{\alpha+1}\right)\left|\frac{\mathrm{d} v_{\alpha}^{\text {all }}}{\mathrm{d} t}\right|^{2} \mathrm{~d} t+\frac{\mathrm{d} u_{\alpha}^{\text {all }}(0)}{\mathrm{d} x} \frac{1}{(\alpha+1)^{\frac{p+2}{p+1}}} \frac{u_{\alpha}^{\text {all }}(0)}{(\alpha+1)^{\frac{1}{p+1}}} \\
& =I^{\text {all }, \alpha}(\alpha+1)^{-\frac{p+3}{p+1}} \int_{0}^{\infty} \exp (-t)\left(v_{\alpha}^{\text {all }}\right)^{p+1} \mathrm{~d} t .
\end{aligned}
$$

Then, it follows that

$$
\begin{aligned}
\int_{0}^{\infty} \exp (-t)\left|\frac{\mathrm{d} v_{\alpha}^{\text {all }}}{\mathrm{d} t}\right|^{2} \mathrm{~d} t & \leqslant \int_{0}^{\infty}\left|\frac{\mathrm{d} v_{\alpha}^{\text {all }}}{\mathrm{d} t}\right|^{2} \mathrm{~d} t \leqslant \int_{0}^{\infty} \exp \left(\frac{t}{\alpha+1}\right)\left|\frac{\mathrm{d} v_{\alpha}^{\text {all }}}{\mathrm{d} t}\right|^{2} \mathrm{~d} t \\
& \leqslant I^{\text {all }, \alpha}(\alpha+1)^{-\frac{p+3}{p+1}}\left\|v_{\alpha}^{\text {all }}\right\|_{L^{\infty}}^{p-1} \int_{0}^{\infty} \exp (-t)\left(v_{\alpha}^{\text {all }}\right)^{2} \mathrm{~d} t .
\end{aligned}
$$


Then, from (4) and (23), we deduce that for $N=1,\left\{\left\|v_{\alpha}^{\text {all }}\right\|_{L^{\infty}}\right\}_{\alpha}$ is bounded away from 0. Prior to proceeding further, we prepare some lemmas.

We consider the $L^{\infty}$ bound first.

Lemma 4.1. For each $N \neq 2,\left\{\left\|v_{\alpha}^{\text {all }}\right\|_{L^{\infty}}\right\}_{\alpha}$ is bounded.

Proof. We prove the claim for the cases $N \geqslant 3$ and $N=1$ separately.

Let $N \geqslant 3$. First of all, we note that for $U_{\alpha} \equiv\left(I^{\text {all }, \alpha}\right)^{\frac{1}{p-1}} u_{\alpha}^{\text {all }}$,

$$
\begin{aligned}
& \Delta U_{\alpha}+\left(U_{\alpha}\right)^{p} \geqslant 0, \quad U_{\alpha}>0 \quad \text { in } B, \\
& U_{\alpha}=0 \text { on } \partial B .
\end{aligned}
$$

Then, by an uniform estimate [2, Proposition 3.5], we see that for $N \geqslant 3$ and some $C>0$, independent of $\alpha$,

$$
\left\|U_{\alpha}\right\|_{L^{\infty}} \leqslant C\left\|U_{\alpha}\right\|_{L^{2 N /(N-2)}}^{4 /(N+2-p(N-2))} .
$$

Thus, from the Sobolev inequality and (20), it follows that for some $C>0$,

$$
\left\|u_{\alpha}^{\mathrm{all}}\right\|_{L^{\infty}}=\left(I^{\mathrm{all}, \alpha}\right)^{-\frac{1}{p-1}}\left\|U_{\alpha}\right\|_{L^{\infty}} \leqslant C\left(I^{\mathrm{all}, \alpha}\right)^{\frac{2}{N+2-p(N-2)}-\frac{1}{p+1}} \leqslant C\left(\frac{\alpha+N}{N}\right)^{\frac{N}{p+1}} .
$$

Thus, for $N \geqslant 3,\left\{\left\|v_{\alpha}^{\text {all }}\right\|_{L^{\infty}}\right\}_{\alpha}$ is bounded.

Let $N=1$. Defining $W_{\alpha} \equiv \frac{\mathrm{d} v_{\alpha}^{\text {all }}}{\mathrm{d} t}$, we see that for $K_{\alpha, 1} \equiv I^{\text {all }, \alpha}(\alpha+1)^{\frac{-(p+3)}{p+1}}$,

$$
\frac{\mathrm{d}^{2} W_{\alpha}}{\mathrm{d} t^{2}}+\frac{\alpha+3}{\alpha+1} \frac{\mathrm{d} W_{\alpha}}{\mathrm{d} t}+\left(\frac{\alpha+2}{(\alpha+1)^{2}}+K_{\alpha, 1} p \exp \left(-\frac{\alpha+2}{\alpha+1} t\right)\left(v_{\alpha}^{\text {all }}\right)^{p-1}\right) W_{\alpha}=0
$$

and

$$
W_{\alpha}(0)>0, \quad \lim _{t \rightarrow \infty} W_{\alpha}(t)=-\lim _{t \rightarrow \infty} \exp \left(-\frac{t}{\alpha+1}\right) \frac{1}{\alpha+1} \frac{\mathrm{d} u_{\alpha}^{\text {all }}(0)}{\mathrm{d} x}=0 .
$$

As in Theorem 3.1, we deduce that for any $a \in(0,1)$, there exists some $C>0$, independent of $\alpha>0$, satisfying

$$
W_{\alpha}(t) \leqslant C \exp (-a t), \quad t \geqslant 0 .
$$

This implies that

$$
v_{\alpha}^{\text {all }}(t)=\int_{0}^{t} W_{\alpha}(s) \mathrm{d} s \leqslant \frac{C}{a}, \quad t>0 .
$$

This completes the proof.

Lemma 4.2. For $N=2$, there exists a constant $C>0$, independent of $\alpha>0$, such that

$$
v_{\alpha}^{\text {all }}(t, \phi) \leqslant C \exp \left(\frac{2 p t}{p^{2}-1}\right), \quad t>0,-\frac{\alpha+2}{2} \pi \leqslant \phi \leqslant \frac{\alpha+2}{2} \pi .
$$

Proof. Let $N=2$ and $K_{\alpha, 2} \equiv I^{\text {all, } \alpha}\left(\frac{2}{\alpha+2}\right)^{\frac{4}{p+1}}$, Then, the $v_{\alpha}^{\text {all }}$ satisfies the following equation

$$
\begin{aligned}
& \frac{\partial^{2} v_{\alpha}^{\text {all }}}{\partial t^{2}}+\frac{\partial^{2} v_{\alpha}^{\text {all }}}{\partial y^{2}}+K_{\alpha, 2} \exp (-2 t)\left(v_{\alpha}^{\text {all }}\right)^{p}=0 \quad \text { in }(0, \infty) \times\left(-\frac{\alpha+2}{2} \pi, \frac{\alpha+2}{2} \pi\right), \\
& \frac{\partial v_{\alpha}^{\text {all }}}{\partial y}=0 \quad \text { on }(0, \infty) \times\left\{-\frac{\alpha+2}{2} \pi, \frac{\alpha+2}{2} \pi\right\}, \\
& v_{\alpha}^{\text {all }}=0 \quad \text { on }\{0\} \times\left(-\frac{\alpha+2}{2} \pi, \frac{\alpha+2}{2} \pi\right) .
\end{aligned}
$$


Let $T>2$, and $\phi$ a smooth function such that

$$
\phi(t, y)= \begin{cases}1 & \text { for } \sqrt{(t-T)^{2}+y^{2}} \leqslant 1 \\ 0 & \text { for } \sqrt{(t-T)^{2}+y^{2}} \geqslant 2\end{cases}
$$

and $0 \leqslant \phi \leqslant 1$ on $\mathbf{R}^{N}$. For the sake of convenience, we denote $v=v_{\alpha}^{\text {all }}$ in the followings. Then, for any $\alpha>0$, multiplying $v^{2 \alpha+1} \phi^{2}$ to above equation for $v$ and integrating by parts, we deduce that

$$
\int\left|\nabla v^{\alpha+1} \phi\right|^{2} \mathrm{~d} t \mathrm{~d} y \leqslant \int v^{2 \alpha+2}|\nabla \phi|^{2} \mathrm{~d} t \mathrm{~d} y+K_{\alpha, 2}(\alpha+1) \int \exp (-2 t) v^{2 \alpha+2} v^{p-1} \phi^{2} \mathrm{~d} t \mathrm{~d} y .
$$

Denoting $w=\exp \left(-\frac{2 t}{p}\right) v \phi^{\frac{2}{p}}$, we see from Hölder's inequality that for any $K>0$,

$$
\begin{aligned}
& \int \exp (-2 t) v^{2 \alpha+2} v^{p-1} \phi^{2} \mathrm{~d} t \mathrm{~d} y=\int w^{p-1} \exp \left(-\frac{2 t}{p}\right) v^{2 \alpha+2} \phi^{\frac{2}{p}} \mathrm{~d} t \mathrm{~d} y \\
& \leqslant\left(\int_{\{(t, y) \mid w(t, y) \geqslant K\}} \exp (-2 t) v^{p} \phi^{2} \mathrm{~d} t \mathrm{~d} y\right)^{\frac{p-1}{p}}\left(\int \exp (-2 t) v^{p(2 \alpha+2)} \phi^{2} \mathrm{~d} t \mathrm{~d} y\right)^{\frac{1}{p}} \\
& \quad+K^{p-1} \int \exp \left(-\frac{2 t}{p}\right) v^{2 \alpha+2} \phi^{\frac{2}{p}} \mathrm{~d} t \mathrm{~d} y .
\end{aligned}
$$

It is easy to see that

$$
\begin{aligned}
& \int_{\{(t, y) \mid w(t, y) \geqslant K\}} \exp (-2 t) v^{p} \phi^{2} \mathrm{~d} t \mathrm{~d} y \\
& \leqslant|\{(t, y) \mid w(t, y) \geqslant K\}|^{\frac{1}{p+1}}\left(\int \exp \left(-2 \frac{(p+1) t}{p}\right) v^{p+1} \phi^{\frac{2(p+1)}{p}} \mathrm{~d} t \mathrm{~d} y\right)^{\frac{p}{p+1}} \\
& \leqslant|\{(t, y) \mid w(t, y) \geqslant K\}|^{\frac{1}{p+1}}\left(\int \exp (-2 t) v^{p+1} \phi^{2} \mathrm{~d} t \mathrm{~d} y\right)^{\frac{p}{p+1}}
\end{aligned}
$$

and

$$
\begin{aligned}
|\{(t, y) \mid w(t, y) \geqslant K\}| & \leqslant K^{-(p+1)} \int \exp \left(-2 \frac{(p+1) t}{p}\right) v^{p+1} \phi^{\frac{2(p+1)}{p}} \mathrm{~d} t \mathrm{~d} y \\
& \leqslant K^{-(p+1)} \int \exp (-2 t) v^{p+1} \phi^{2} \mathrm{~d} t \mathrm{~d} y .
\end{aligned}
$$

Thus, it follows that

$$
\int_{\{(t, y) \mid w(t, y) \geqslant K\}} \exp (-2 t) v^{p} \phi^{2} \mathrm{~d} t \mathrm{~d} y \leqslant \frac{1}{K} \int \exp (-2 t) v^{p+1} \phi^{2} \mathrm{~d} t \mathrm{~d} y .
$$

Combining (26)-(29) and Proposition 2.1, we see that for some $C>0$, independent of $\alpha, \phi$ and $v$,

$$
\begin{aligned}
& \left(\int \exp (-2 t) v^{p(2 \alpha+2)} \phi^{2 p} \mathrm{~d} t \mathrm{~d} y\right)^{\frac{1}{p}} \\
& \leqslant C \int v^{2 \alpha+2}|\nabla \phi|^{2} \mathrm{~d} t \mathrm{~d} y++C(\alpha+1) K^{p-1} \int \exp \left(-\frac{2 t}{p}\right) v^{2 \alpha+2} \phi^{\frac{2}{p}} \mathrm{~d} t \mathrm{~d} y \\
& \quad+C(\alpha+1)\left(\frac{1}{K} \int \exp (-2 t) v^{p+1} \phi^{2} \mathrm{~d} t \mathrm{~d} y\right)^{\frac{p-1}{p}}\left(\int \exp (-2 t) v^{p(2 \alpha+2)} \phi^{2} \mathrm{~d} t \mathrm{~d} y\right)^{\frac{1}{p}} .
\end{aligned}
$$

We take $K>0$ so that

$$
C(\alpha+1)\left(\frac{1}{K} \int \exp (-2 t) v^{p+1} \phi^{2} \mathrm{~d} t \mathrm{~d} y\right)^{\frac{p-1}{p}}=\frac{1}{2} .
$$


Then, since

$$
\int_{-\frac{(\alpha+N) \pi}{N}}^{\frac{(\alpha+N) \pi}{N}} \int_{0}^{\infty} \exp (-2 t) v^{p+1} \mathrm{~d} t \mathrm{~d} y=1,
$$

it follows that

$$
\begin{aligned}
& \left(\int \exp (-2 t) v^{p(2 \alpha+2)} \phi^{2 p} \mathrm{~d} t \mathrm{~d} y\right)^{\frac{1}{p}} \\
& \leqslant 2 C \int v^{2 \alpha+2}|\nabla \phi|^{2} \mathrm{~d} t \mathrm{~d} y+(2 C(\alpha+1))^{1-p}\left(\int \exp (-2 t) v^{p+1} \phi^{2} \mathrm{~d} t \mathrm{~d} y\right)^{p-1} \int \exp \left(-\frac{2 t}{p}\right) v^{2 \alpha+2} \phi^{\frac{2}{p}} \mathrm{~d} t \mathrm{~d} y \\
& \leqslant 2 C \int v^{2 \alpha+2}|\nabla \phi|^{2} \mathrm{~d} t \mathrm{~d} y+(2 C(\alpha+1))^{1-p} \int \exp \left(-\frac{2 t}{p}\right) v^{2 \alpha+2} \phi^{\frac{2}{p}} \mathrm{~d} t \mathrm{~d} y .
\end{aligned}
$$

We take a smooth function $\phi_{i}$ such that

$$
\phi_{i}(t, y)= \begin{cases}1 & \text { for } \sqrt{(T-t)^{2}+y^{2}} \leqslant 1+2^{-i} \\ 0 & \text { for } \sqrt{(T-t)^{2}+y^{2}} \geqslant 1+2^{-i+1}\end{cases}
$$

and $0 \leqslant \phi_{i} \leqslant 1,\left|\nabla \phi_{i}\right| \leqslant 2^{i+1}$. Then, substituting $\phi$ and $2 \alpha+2$ by $\phi_{i}$ and $(p+1) p^{i-1}$ respectively in above inequality, we see that

$$
\begin{aligned}
& \left(\int_{B\left((T, 0), 1+2^{i}\right)} \exp (-2 t) v^{p^{i}(p+1)} \mathrm{d} t \mathrm{~d} y\right)^{\frac{1}{p}} \\
& \leqslant 2 C 4^{i+1} \exp (2 T+4) \int_{B\left((T, 0), 1+2^{i-1}\right)} \exp (-2 t) v^{p^{i-1}(p+1)} \mathrm{d} t \mathrm{~d} y \\
& \quad+(2 C(\alpha+1))^{1-p} \exp \left(\frac{p-1}{p}(2 T+4)\right) \int_{B\left((T, 0), 1+2^{i-1}\right)} \exp (-2 t) v^{p^{i-1}(p+1)} \mathrm{d} t \mathrm{~d} y .
\end{aligned}
$$

Then, we deduce that for some $D>0$, independent of $i$ and $T>0$,

$$
\begin{aligned}
& \left(\int_{B\left((T, 0), 1+2^{i}\right)} \exp (-2 t) v^{p^{i}(p+1)} \mathrm{d} t \mathrm{~d} y\right)^{\frac{1}{p^{i}(p+1)}} \\
& \leqslant D \exp \left(2 T \sum_{j=1}^{i} \frac{1}{p^{j-1}(p+1)}\right)\left(\int_{B((T, 0), 2)} \exp (-2 t) v^{p+1} \mathrm{~d} t \mathrm{~d} y\right)^{\frac{1}{p+1}} \\
& \leqslant D \exp \left(2 T \frac{p}{p^{2}-1}\right) .
\end{aligned}
$$

Then, taking $i \rightarrow \infty$ in above inequality, we see that for some $D>0$,

$$
v(T, 0) \leqslant D \exp \left(2 T \frac{p}{p^{2}-1}\right), \quad T>2 .
$$

This proves the claim.

Lemma 4.3. Let $N \geqslant 3$, and let $A_{\alpha}:=\lim _{t \rightarrow \infty} v_{\alpha}^{\text {all }}(t, \phi)$. Then $\lim _{\alpha \rightarrow \infty} A_{\alpha}=0$. 
Proof. Denoting $U_{\alpha} \equiv\left(I^{\text {all }, \alpha}\right)^{1 /(p-1)} u_{\alpha}^{\text {all }}$, we see that

$$
\Delta U_{\alpha}+|x|^{\alpha}\left(U_{\alpha}\right)^{p}=0 \quad \text { in } B(0,1),
$$

and that

$$
\int_{B(0,1)}|x|^{\alpha}\left(U_{\alpha}\right)^{p+1}(x) \mathrm{d} x=\left(I^{\mathrm{all}, \alpha}\right)^{\frac{p+1}{p-1}} .
$$

Let $G(x, y)$ be the Green function of $-\Delta$ on $B(0,1)$. Then, we see that

$$
U_{\alpha}(x)=-\int_{B(0,1)} G(y, x)|y|^{\alpha}\left(U_{\alpha}\right)^{p}(y) \mathrm{d} y .
$$

Note that $G(x, 0)=C\left(1 /|x|^{N-2}-1\right)$ for some $C>0$. Applying Hölder's inequality, we deduce that for some constant $C>0$,

$$
\begin{aligned}
U_{\alpha}(0) & \leqslant C\left(\int_{B(0,1)}(G(x, 0))^{p+1}|x|^{\alpha} \mathrm{d} x\right)^{1 /(p+1)}\left(\int_{B(0,1)}|x|^{\alpha}\left(U_{\alpha}\right)^{p+1} \mathrm{~d} x\right)^{p /(p+1)} \\
& \leqslant C(\alpha+C)^{-1 /(p+1)}\left(I^{\mathrm{all}, \alpha}\right)^{p /(p-1)}
\end{aligned}
$$

Thus, it follows that

$$
\begin{aligned}
\lim _{t \rightarrow \infty} v_{\alpha}^{\text {all }}(t, \phi) & =\left(\frac{N}{\alpha+N}\right)^{\frac{N}{p+1}} u_{\alpha}^{\text {all }}(0, \theta) \leqslant C I^{\text {all }, \alpha}\left(\frac{N}{\alpha+N}\right)^{\frac{N}{p+1}+\frac{1}{p+1}} \\
& \leqslant C\left(\frac{\alpha+N}{N}\right)^{\frac{N+2-p(N-2)}{p+1}-\frac{N+1}{p+1}}=C\left(\frac{\alpha+N}{N}\right)^{\frac{1-p(N-2)}{p+1}} .
\end{aligned}
$$

Since $\frac{1-p(N-2)}{p+1}<0$ for $p>1$ and $N \geqslant 3$, the claim follows.

Lemma 4.4. Let $N \geqslant 3$. Suppose that there exists $T_{\alpha}>0$ satisfying $\lim _{\alpha \rightarrow \infty} T_{\alpha}=\infty$ and

$$
\lim _{\alpha \rightarrow \infty} \sup _{\phi \in S_{\alpha}^{N-1}} v_{\alpha}^{\text {all }}\left(T_{\alpha}, \phi\right)=0 .
$$

Then, it follows that

$$
\lim _{\alpha \rightarrow \infty} \sup \left\{v_{\alpha}^{\text {all }}(t, \phi) \mid t \geqslant T_{\alpha}, \phi \in S_{\alpha}^{N-1}\right\}=0 .
$$

Proof. Let $\left(\phi_{\frac{N}{2}, 1}, \lambda_{\frac{N}{2}, 1}\right)$ be a pair of the first eigenfunction and the first eigenvalue of

$$
\begin{aligned}
& \frac{\mathrm{d}^{2} \phi}{\mathrm{d} t^{2}}+\lambda \exp \left(-\frac{N t}{2}\right) \phi=0 \quad \text { on }(0, \infty), \\
& \phi(0)=0, \\
& \phi \in H((0, \infty))
\end{aligned}
$$

satisfying that for $t>0, \phi_{\frac{N}{2}, 1}(t)>0$, and $\lim _{t \rightarrow \infty} \phi_{\frac{N}{2}, 1}(t)=1$. Note that $\frac{\mathrm{d} \phi_{1}(t)}{\mathrm{d} t}>0$ for $t>0$. Let $K_{\alpha, N} \equiv$ $I^{\text {all }, \alpha}\left(\frac{N}{\alpha+N}\right)^{\frac{N+2-(N-2) p}{p+1}}$. From the boundedness of $\left\{\left\|v_{\alpha}^{\text {all }}\right\|_{L^{\infty}}\right\}_{\alpha}$ for $N \geqslant 3$, we see that for sufficiently large $T>0$,

$$
\begin{aligned}
& \left(\phi_{\frac{N}{2}, 1}\right)_{t t}-\frac{N(N-2)}{\alpha+N}\left(\phi_{\frac{N}{2}, 1}\right)_{t}+\Delta_{S_{\alpha}^{N-1}} \phi_{\frac{N}{2}, 1}+K_{\alpha, N} \exp \left(-\frac{N(\alpha+2) t}{\alpha+N}\right)\left(v_{\alpha}^{\text {all }}\right)^{p-1} \phi_{\frac{N}{2}, 1} \\
& \quad \leqslant \phi_{\frac{N}{2}, 1} \exp \left(-\frac{N(\alpha+2) t}{\alpha+N}\right)\left(K_{\alpha, N}\left(v_{\alpha}^{\text {all }}\right)^{p-1}-\exp \left(\frac{N(\alpha+4-N) t}{2 \alpha+2 N}\right) \lambda_{\frac{N}{2}, 1}\right) \leqslant 0, \quad t \geqslant T .
\end{aligned}
$$


From a comparison principle (refer [12]), (22) and Lemma 4.3, we deduce as in Proposition 2.3 that

$$
\lim _{\alpha \rightarrow \infty} \sup \left\{v_{\alpha}^{\text {all }}(t, \phi) \mid t \geqslant T_{\alpha}, \phi \in S_{\alpha}^{N-1}\right\}=0 .
$$

This completes the proof of Lemma 4.4.

Now we consider the limit of $v_{\alpha}^{\text {all }}$. Note that for each $T>0$,

$$
\int_{(0, T) \times S_{\alpha}^{N-1}}\left(v_{\alpha}^{\text {all }}\right)^{p+1} \mathrm{~d}_{\alpha} \sigma \mathrm{d} t \leqslant \exp (N T) .
$$

Then, from elliptic estimates [8], we deduce that there exists $\gamma \in(0,1)$ such that $\left\{\left|v_{\alpha}^{\text {all }}\right|_{C^{2, \gamma}\left((0, T) \times S_{\alpha}^{N-1}\right)}\right\}_{\alpha}$ is bounded for any $T<\infty$. Thus, for some $K \in\left[0, J_{N, N}\right], v_{\alpha}(t, y)=v_{\alpha}^{\text {all }}\left(t, \psi_{\alpha}(y)\right)$ converges in $C_{\text {loc }}^{2}$ to some $w$ satisfying

$$
\begin{aligned}
& \Delta w+K \exp (-N t) w^{p}=0 \text { in }(0, \infty) \times \mathbf{R}^{N-1}, \\
& w=0 \quad \text { on }\{0\} \times \mathbf{R}^{N-1} .
\end{aligned}
$$

Furthermore, it follows that

$$
\int_{(0, \infty) \times \mathbf{R}^{N-1}}|\nabla w|^{2} \mathrm{~d} t \mathrm{~d} y \leqslant J_{N, N} \quad \text { and } \int_{(0, \infty) \times \mathbf{R}^{N-1}} \exp (-N t) w^{p+1} \mathrm{~d} t \mathrm{~d} y \leqslant 1 .
$$

Then, we see the following result.

Lemma 4.5. For each $N \geqslant 1, w>0$ in $(0, \infty) \times \mathbf{R}^{N-1}$.

Proof. To the contrary, suppose that $w=0$.

First, consider the cases $N \geqslant 3$. From Lemma 4.1, we see that

$$
\lim _{\alpha \rightarrow \infty}\left\|\exp \left(-\frac{N t}{2}\right) v_{\alpha}^{\text {all }}\right\|_{L^{\infty}\left((0, \infty) \times S_{\alpha}\right)}=0 .
$$

Then, for sufficiently large $\alpha>0$ and $N>2$, we see that

$$
\begin{aligned}
& \int_{(0, \infty) \times S_{\alpha}^{N-1}} \exp \left(-\frac{N t}{2}\right)\left(\left|\frac{\partial v_{\alpha}^{\text {all }}}{\partial t}\right|^{2}+\left|\nabla_{S_{\alpha}} v_{\alpha}^{\text {all }}\right|^{2}\right) \mathrm{d} t \mathrm{~d}_{\alpha} \sigma \\
\leqslant & \int_{(0, \infty) \times S_{\alpha}^{N-1}} \exp \left(-\frac{N(N-2) t}{\alpha+N}\right)\left(\left|\frac{\partial v_{\alpha}^{\text {all }}}{\partial t}\right|^{2}+\left|\nabla_{S_{\alpha}} v_{\alpha}^{\text {all }}\right|^{2}\right) \mathrm{d} t \mathrm{~d}_{\alpha} \sigma \\
= & K_{\alpha, N} \int_{(0, \infty) \times S_{\alpha}^{N-1}} \exp (-N t)\left(v_{\alpha}^{\text {all }}\right)^{p+1} \mathrm{~d} t \mathrm{~d}_{\alpha} \sigma \\
\leqslant & K_{\alpha, N}\left\|\exp \left(-\frac{N t}{2}\right)\left(v_{\alpha}^{\text {all }}\right)^{p-1}\right\|_{L^{\infty}\left((0, \infty) \times S_{\alpha}\right)} \int_{(0, \infty) \times S_{\alpha}^{N-1}} \exp \left(-\frac{N}{2} t\right)\left(v_{\alpha}^{\text {all }}\right)^{2} \mathrm{~d} t \mathrm{~d}_{\alpha} \sigma,
\end{aligned}
$$

where $K_{\alpha, N}=I^{\text {all }, \alpha}\left(\frac{\alpha+N}{N}\right)^{\frac{N+2-(N-2) p}{p+1}}$. On the other hand, integrating both sides of (4) on $S_{\alpha}$ with respect to $y$, we see that for some $C>0$,

$$
\int_{(0, \infty) \times S_{\alpha}^{N-1}} \exp \left(-\frac{N}{2} t\right)\left(v_{\alpha}^{\text {all }}\right)^{2} \mathrm{~d} t \mathrm{~d}_{\alpha} \sigma \leqslant C \int_{(0, \infty) \times S_{\alpha}^{N-1}} \exp \left(-\frac{N t}{2}\right)\left(\left|\frac{\partial v_{\alpha}^{\text {all }}}{\partial t}\right|^{2}+\left|\nabla_{S_{\alpha}} v_{\alpha}^{\text {all }}\right|^{2}\right) \mathrm{d} t \mathrm{~d}_{\alpha} \sigma .
$$

Since $\lim _{\alpha \rightarrow \infty} K_{\alpha, N} \leqslant J_{N, N}$, this is a contradiction. 
Secondly, consider the case $N=2$. Note that a function $\psi=\partial v_{\alpha}^{\text {all }} / \partial \phi$ satisfies

$$
\begin{aligned}
& \Delta \psi+K_{\alpha, 2} \exp (-2 t)\left(v_{\alpha}^{\mathrm{all}}\right)^{p-1} \psi=0 \quad \text { in }(0, \infty) \times\left(-\frac{\alpha+N}{N} \pi, \frac{\alpha+N}{N} \pi\right), \\
& \psi=0 \quad \text { on } \partial\left((0, \infty) \times\left(-\frac{\alpha+N}{N} \pi, \frac{\alpha+N}{N} \pi\right)\right) .
\end{aligned}
$$

It is obvious that

$$
\lim _{t \rightarrow \infty} \sup \left\{\psi(t, y) \mid y \in\left(-\frac{\alpha+N}{N} \pi, \frac{\alpha+N}{N} \pi\right)\right\}=0 .
$$

From Lemma 4.2, we see that

$$
\begin{aligned}
& \lim _{t \rightarrow \infty} \sup \left\{\exp (-2 t)\left(v_{\alpha}^{\text {all }}\right)^{p-1}(t, y) \mid y \in\left(-\frac{\alpha+N}{N} \pi, \frac{\alpha+N}{N} \pi\right)\right\} \\
& =\lim _{t \rightarrow \infty} \sup \left\{\exp \left(-\frac{(2 p+1) t}{p+1}\right)\left(v_{\alpha}^{\text {all }}\right)^{p-1}(t, y) \mid y \in\left(-\frac{\alpha+N}{N} \pi, \frac{\alpha+N}{N} \pi\right)\right\}=0 .
\end{aligned}
$$

that a set $\left\{\|\psi\|_{C^{2}}\right\}$ is bounded. Then, by integration by parts, we see that

$$
\int_{0}^{\infty} \int_{-\frac{\alpha+N}{N} \pi}^{\frac{\alpha+N}{N} \pi}|\nabla \psi|^{2} \mathrm{~d} t \mathrm{~d} y=K_{\alpha, 2} \int_{0}^{\infty} \int_{-\frac{\alpha+N}{N} \pi}^{\frac{\alpha+N}{N} \pi} \exp (-2 t)\left(v_{\alpha}^{\mathrm{all}}\right)^{p-1} \psi^{2} \mathrm{~d} t \mathrm{~d} y<\infty .
$$

Thus, it follows that $\psi \in H((0, \infty) \times \mathbf{R})$. Moreover, since

$$
\int_{0}^{\infty} \int_{-\frac{\alpha+N}{N} \pi}^{\frac{\alpha+N}{N} \pi} \exp (-2 t)\left(v_{\alpha}^{\mathrm{all}}\right)^{p-1} \psi^{2} \mathrm{~d} t \mathrm{~d} y \leqslant\left\|\exp \left(-\frac{(2 p+1) t}{p+1}\right)\left(v_{\alpha}^{\mathrm{all}}\right)^{p-1}\right\|_{L^{\infty}} \int_{0}^{\infty} \int_{-\frac{\alpha+N}{N} \pi}^{\frac{\alpha+N}{N} \pi} \exp \left(-\frac{t}{p+1}\right) \psi^{2} \mathrm{~d} t \mathrm{~d} y,
$$

it follows that

$$
\int_{0}^{\infty} \int_{-\frac{\alpha+N}{N} \pi}^{\frac{\alpha+N}{N} \pi}|\nabla \psi|^{2} \mathrm{~d} t \mathrm{~d} y \leqslant K_{\alpha, 2}\left\|\exp \left(-\frac{(2 p+1) t}{p+1}\right)\left(v_{\alpha}^{\mathrm{all}}\right)^{p-1}\right\|_{L^{\infty}} \int_{0}^{\infty} \int_{-\frac{\alpha+N}{N} \pi}^{\frac{\alpha+N}{N} \pi} \exp \left(-\frac{t}{p+1}\right) \psi^{2} \mathrm{~d} t \mathrm{~d} y .
$$

Note that $\lim _{\alpha \rightarrow \infty} K_{\alpha, 2} \leqslant J_{2,2}$ and

$$
\lim _{\alpha \rightarrow \infty}\left\|\exp \left(-\frac{(2 p+1) t}{p+1}\right)\left(v_{\alpha}^{\text {all }}\right)^{p-1}\right\|_{L^{\infty}}=0 .
$$

This contradicts (5).

Finally, we consider the case $N=1$. As in (25), we deduce that

$$
\int_{0}^{\infty} \exp \left(-\frac{t}{2}\right)\left|\frac{\mathrm{d} v_{\alpha}^{\text {all }}}{\mathrm{d} t}\right|^{2} \mathrm{~d} t \leqslant \int_{0}^{\infty} \exp \left(\frac{t}{\alpha+1}\right)\left|\frac{\mathrm{d} v_{\alpha}^{\text {all }}}{\mathrm{d} t}\right|^{2} \mathrm{~d} t \leqslant K_{\alpha, 1}\left\|\exp \left(-\frac{t}{2}\right) v_{\alpha}^{\text {all }}\right\|_{L^{\infty}}^{p-1} \int_{0}^{\infty} \exp \left(-\frac{t}{2}\right)\left(v_{\alpha}^{\text {all }}\right)^{2} \mathrm{~d} t .
$$

Since $w=0, \lim _{\alpha \rightarrow \infty} K_{\alpha, 1} \leqslant J_{1,1}$ and $\left\{\left\|v_{\alpha}^{\text {all }}\right\|_{L^{\infty}}\right\}_{\alpha}$ is bounded, it follows that

$$
\lim _{\alpha \rightarrow \infty} K_{\alpha, 1}\left\|\exp \left(-\frac{t}{2}\right)\left(v_{\alpha}^{\text {all }}\right)^{p-1}\right\|_{L^{\infty}}=0 .
$$

This contradicts (4).

Therefore, we conclude that $w>0$ in $(0, \infty) \times \mathbf{R}^{N-1}$. The proof of Lemma 4.5 is finished. 
If $K=0$ in (30), the limit function $w$ is harmonic. Then, it is easy to see that $w=a t$ for some $a>0$. This contradicts that $\int_{0}^{\infty} \int_{\mathbf{R}^{N-1}}|\nabla w| \mathrm{d} t \mathrm{~d} y \leqslant \infty$. Thus, we have $K>0$. Now, let $\gamma=\int_{0}^{\infty} \int_{\mathbf{R}^{N-1}} \exp (-N t) w^{p+1} \mathrm{~d} t \mathrm{~d} y \in$ $(0,1]$ and $W=\gamma^{-1 /(p+1)} w$. Then, we see that $\int_{0}^{\infty} \int_{\mathbf{R}^{N-1}} \exp (-N t) W^{p+1} \mathrm{~d} t \mathrm{~d} y=1$, and that

$$
\Delta W+K \gamma^{(p-1) /(p+1)} \exp (-N t) W^{p}=0 \quad \text { in }(0, \infty) \times \mathbf{R}^{N-1} .
$$

This implies that $\int_{0}^{\infty} \int_{\mathbf{R}^{N-1}}|\nabla W|^{2} \mathrm{~d} t \mathrm{~d} y=K \gamma^{(p-1) /(p+1)}$. Thus, it follows that

$$
K \gamma^{(p-1) /(p+1)} \geqslant J_{N, N}
$$

Since $J_{N, N} \geqslant K$ by (20) and $K \geqslant K \gamma^{(p-1) /(p+1)}$, it follows that $K=J_{N, N}$ and $\gamma=1$. Therefore, the function $w$ is a minimizer of $J_{N, N}$, and

$$
\lim _{\alpha \rightarrow \infty} I^{\mathrm{all}, \alpha}\left(\frac{N}{\alpha+N}\right)^{\frac{N+2-(N-2) p}{p+1}}=J_{N, N} .
$$

To complete the proof of Theorem 4.1, it suffices to show that $V_{\alpha}^{\text {all }}$ (defined in the statement of Theorem 4.1) converges uniformly to $w$ for $N \geqslant 3$. It is standard to see that for each $T>0, \lim _{|y|, \alpha \rightarrow \infty} V_{\alpha}^{\text {all }}(t, y)=0$ uniformly for $t \in(0, T)$. Note that $\lim _{\alpha \rightarrow \infty} \sup _{\phi \in S_{\alpha}^{N-1}} v_{\alpha}^{\text {all }}(T, \phi) \leqslant w(T, 0)$. Then, since $\lim _{t \rightarrow \infty} \sup _{y \in \mathbf{R}^{N-1}} w(t, y)=0$ for $N \geqslant 3$ (Proposition 2.5), by Lemma 4.4,

$$
\lim _{t \rightarrow \infty, \alpha \rightarrow \infty} \sup _{y \in \mathbf{R}^{N-1}} V_{\alpha}^{\text {all }}(t, y)=0 .
$$

Thus, $V_{\alpha}^{\text {all }}(t, y)$ converges uniformly to $w$ for $N \geqslant 3$. For the convergence of $v_{\alpha}^{\text {all, }-} \equiv\left(\frac{1}{\alpha+1}\right)^{1 /(p+1)} u_{\alpha}^{\text {all }}\left(-\exp \left(-\frac{t}{\alpha+1}\right)\right)$, we note that

$$
1=\int_{0}^{\infty} \exp (-t) w^{p+1}=\lim _{\alpha \rightarrow \infty} \int_{0}^{\infty} \exp (-t)\left(v_{\alpha}^{\mathrm{all}}\right)^{p+1} \mathrm{~d} t=\lim _{\alpha \rightarrow \infty} \int_{0}^{1}|x|^{\alpha}\left(u_{\alpha}^{\mathrm{all}}\right)^{p+1} \mathrm{~d} x
$$

and

$$
\int_{-1}^{1}|x|^{\alpha}\left(u_{\alpha}^{\mathrm{all}}\right)^{p+1} \mathrm{~d} x=\int_{0}^{\infty} \exp (-t)\left(v_{\alpha}^{\mathrm{all}}\right)^{p+1} \mathrm{~d} t+\int_{0}^{\infty} \exp (-t)\left(v_{\alpha}^{\mathrm{all},-}\right)^{p+1} \mathrm{~d} t .
$$

Thus,

$$
\lim _{\alpha \rightarrow \infty} \int_{0}^{\infty} \exp (-t)\left(v_{\alpha}^{\mathrm{all},-}\right)^{p+1} \mathrm{~d} t=0
$$

Then, the convergence of $v_{\alpha}^{\text {all,- }}$ comes from standard elliptic estimates [8]. This completes the proof of Theorem 4.1 .

\section{Some final remarks}

First, as a corollary of Theorems 3.1, 3.1-E, 4.1 and 4.1-E, we obtain symmetry breaking of least energy solutions of the Hénon equation (1). For $N \geqslant 2$, this was proved in [14] by a different argument.

Corollary 5.1. For $N \geqslant 1$ and $p \in\left(1,2^{*}-1\right)$ fixed, a minimizer $u_{\alpha}^{\text {all }}$ of $I^{\text {all, } \alpha}$ and a least energy solution $\omega_{\alpha}^{\text {all }}$ of (1) is not radially symmetric if $\alpha>0$ is sufficiently large.

As it can be seen in Theorems 3.1 and 3.1-E, the behaviour of $u_{\alpha}^{\mathrm{rad}}$ and $\omega_{\alpha}^{\mathrm{rad}}$ as $\alpha \rightarrow \infty$ is rather completely understood. On the other hand, the behaviour of nonradial ground states $u_{\alpha}^{\text {all }}$ and $\omega_{\alpha}^{\text {all }}$ as $\alpha \rightarrow \infty$ is not quite completely understood. The following questions are worth further study. 
1. What is the exact growth rate of $u_{\alpha}^{\text {all }}(0)$ for $N \geqslant 2$ as $\alpha \rightarrow \infty$ ? Through the proof of Theorem 4.1, we showed that if $N \geqslant 3$, there exists some constant $C>0$ satisfying

$$
\left(\frac{\alpha+N}{N}\right)^{\frac{-N}{p+1}} u_{\alpha}^{\text {all }}(0) \leqslant C\left(\frac{\alpha+N}{N}\right)^{\frac{1-p(N-2)}{p+1}} \rightarrow 0 \quad \text { as } \alpha \rightarrow 0
$$

and

$$
\frac{1}{C} \leqslant \lim \left(\frac{\alpha+N}{N}\right)^{\frac{-N}{p+1}} \max _{x \in B(0,1)} u_{\alpha}^{\text {all }}(x) \leqslant C .
$$

From the Harnack inequality, we see that for any fixed $x \in B(0,1)$, the growth rate of $u_{\alpha}^{\text {all }}(x)$ is the same with that of $u_{\alpha}^{\text {all }}(0)$.

2. Another question is whether a finer convergence of $u_{\alpha}^{\text {all }}$ for $N=2$ hold. The main difficulty for $N=2$ comes from the fact that there is no appropriate inequality of Sobolev type which is independent of domains.

3. There is a unique maximum point $x_{\alpha}$ of $u_{\alpha}^{\text {all }}$ for $N=1$. It would be interesting to know the asymptotic behaviour of $x_{\alpha}$ as $\alpha \rightarrow \infty$.

\section{Note added in proof}

After the submission of this paper, there have been several works in relation to the problems studied in this paper: [15] for symmetry of ground states using polarization method, [5,4,13] for critical or near critical exponent problems, and our work [3] in sequel to the current paper for limiting ground states in general bounded domains.

\section{Acknowledgements}

This work was done through the first author's visit to the Mathematics Department of Utah State University in July, 2001 and January, 2002. The first author would like to express his thanks for their invitation and hospitality. The first author was also supported by Grant No. 1999-2-102-003-5 from the Interdisciplinary Research Program of the KOSEF.

\section{References}

[1] H. Brezis, Symmetry in nonlinear PDE's, in: Differential Equations: La Pietra 1996 (Florence), in: Proc. Sympos. Pure Math., vol. 65, Amer. Math. Soc., Providence, 1999, pp. 1-12.

[2] J. Byeon, Existence of large positive solutions of some nonlinear elliptic equations on singularly perturbed domains, Comm. Partial Differential Equations 22 (1997) 1731-1769.

[3] J. Byeon, Z.-Q. Wang, On the Hénon equation: asymptotic profile of ground states, II, J. Differential Equations 216 (2005) 78-108.

[4] D. Cao, S. Peng, The asymptotic behavior of the ground state solutions for Hénon equation, J. Math. Anal. Appl. 278 (2003) 1-17.

[5] D. Cao, S. Peng, S. Yan, Asymptotic behavior of ground state solutions for the Hénon equation.

[6] G. Chen, J. Zhou, W.M. Ni, Algorithms and visualization for solutions of nonlinear elliptic equations, Int. J. Bifurcation and Chaos 10 (1997) 1731-1769.

[7] B. Gidas, W.N. Ni, L. Nirenberg, Symmetry and related properties via the maximum principle, Comm. Math. Phys. 68 (1979) $209-243$.

[8] D. Gilbarg, N. Trudinger, Elliptic Partial Differential Equations of Second Order, Springer-Verlag, 1983.

[9] M. Hénon, Numerical experiments on the stability of spherical stellar systems, Astronom. Astrophys. 24 (1973) $229-238$.

[10] B. Kawohl, Rearrangements and Convexity of Level Sets in PDE, Lecture Notes in Math., vol. 1150, Springer-Verlag, Berlin, 1985.

[11] W.M. Ni, A nonlinear Dirichlet problem on the unit ball and its applications, Indiana Univ. Math. J. 31 (1982) $801-807$.

[12] H. Protter, H.F. Weinberger, Maximum Principles in Differential Equations, Springer-Verlag, New York, 1984.

[13] E. Serra, Non radial positive solutions for the Hénon equation with critical growth, Calc. Var. Partial Differential Equations 23 (2005) $301-326$.

[14] D. Smets, J. Su, M. Willem, Non-radial ground states for the Hénon equation, Comm. Contemp. Math. 4 (2002) $467-480$.

[15] D. Smets, M. Willem, Partial symmetry and asymptotic behavior for some elliptic variational problems, Calc. Var. Partial Differential Equations 18 (2003) 57-75. 